\title{
NIR Laser Responsive Nanoparticles for Ovarian Cancer Targeted Combination Therapy with Dual-Modal Imaging Guidance
}

\author{
Jiawen Zhao (D) \\ Liang Zhang ${ }^{2}$ \\ Yingjie $\mathrm{Qi}^{3}$ \\ Kui Liao ${ }^{4}$ \\ Zhigang Wang $\mathbb{D}^{5}$ \\ Ming Wen' \\ Di Zhou'
}

'Department of Radiology, The First Affiliated Hospital of Chongqing Medical University, Chongqing, People's Republic of China; ${ }^{2}$ Department of Ultrasound, The First Affiliated Hospital of Chongqing Medical University, Chongqing, People's Republic of China; ${ }^{3}$ Department of Intensive Care Unit (ICU), Dianjiang People's Hospital of Chongqing, Chongqing, People's Republic of China; ${ }^{4}$ Department of Oncology, The First Affiliated Hospital of Chongqing Medical University, Chongqing, People's Republic of China; ${ }^{5}$ Institute of Ultrasound Imaging, The Second Affiliated Hospital of Chongqing Medical University, Chongqing, People's Republic of China

Correspondence: Di Zhou Department of Radiology, The First Affiliated Hospital of Chongqing Medical University, Chongqing, 400010, People's

Republic of China

Tel +8613508329306

Email zhoudi@cqmu.edu.cn
Purpose: Multifunctional nanoparticles with targeted therapeutic function and diagnosticimaging are of great interest in the domain of precision therapy. NIR laser responsive nanoparticles (PLGA-PEG-FA encapsulating $\mathrm{Bi}_{2} \mathrm{~S}_{3}$, PFP, and Dox (designed as FBPD NPs)) are synthesized for ovarian cancer targeted combination therapy with CT/PA dualmodal imaging guidance (PA: photoacoustic; CT: X-ray computed tomography).

Methods and Results: The FBPD NPS prepared by the double emulsification method revealed excellent dispersity, great stability, outstanding optical properties. The temperature of FBPD NPs increased rapidly after laser irradiation, inducing liquid-to-gas conversion of perfluoropentane (PFP), and promoting the release of Dox up to $86.7 \%$. These FBPD NPs demonstrated their outstanding imaging capability for both PA and CT imaging both in vitro and in vivo, providing the potential for therapeutic guidance and monitoring. Assisted by folic acid, these nanoparticles could highly enrich in ovarian tumor tissue and the accumulation peaked at $3 \mathrm{~h}$ after intravenous administration. The desirable photothermal-conversion efficiency of the nanoparticles combined with chemotherapy achieved highly efficient therapy, which was demonstrated both in vitro and in vivo.

Conclusion: We successfully constructed multifunctional theranostic FBPD NPs for highly efficient PTT/chemotherapy combined therapy with dual CT/PA imaging guidance/monitoring. The unique nanoparticles with multiple abilities pave an emerging way toward precise treatment of ovarian cancer.

Keywords: bismuth sulfide, folic acid, chemotherapy, photothermal therapy

\section{Introduction}

Ovarian cancer is the deadliest gynecological malignancy and one of the main causes of cancer death among women. ${ }^{1,2}$ According to American Cancer Society (ACS), it is estimated that there are around 22,240 new diagnoses of ovarian cancer and 14,070 deaths every year. No obvious symptoms and the lack of specific screening method in the early stage of ovarian cancer leads to the lethality. Thus, ovarian cancer is usually diagnosed in the advanced stages (stage III and IV) and the five-year survival rate declines substantially $(39 \%$ for stage III and $17 \%$ for stage IV). ${ }^{3}$ Although conventional treatments, including surgery, chemotherapy, radiotherapy, etc., have proved to be efficient, they are often associated with certain unignorable weaknesses, such as immune system depletion, high cost, patient nonadherence, tumor recurrence and drug resistance. ${ }^{4-7}$ These clinical problems have accordingly heightened the need for more specific and effective tumor 
treatment. These potential therapeutic strategies should meet the rigorous demands of precise killing effect without damaging normal cells/tissues. ${ }^{8}$

Among all the traditional treatments, chemotherapy is the most commonly used method for tumor treatment. ${ }^{5,9}$ As one of the most widespread anthracycline anti-cancer drugs, doxorubicin (Dox), as an ordinary Food and Drug Administration (FDA) approved anti-cancer drug, kills tumor cells through a series of nuclear events, including DNA damage and apoptosis. ${ }^{10,11}$ However, the clinical application of Dox remains challenging due to its low aqueous solubility, inadequate accumulation, and nonspecific distribution. ${ }^{12}$ In the meanwhile, the systemic medication is always inevitably accompanied by various side effects, such as cardiac toxicity, gastrointestinal, and bone marrow suppression. ${ }^{13}$ In order to mitigate the aforementioned adverse effects, drugs are encapsulated by nanoparticles for precise delivery owing to their incomparable advantages such as non-toxicity and enhanced biocompatibility. ${ }^{14,15}$ More importantly, nanoparticles have been proved to boost drug solubility significantly and minimize the systematic toxicity of traditional antitumor agents, strengthening the therapeutic index ultimately. ${ }^{16}$ Poly lactic-co-glycolic acid (PLGA) is a widely used FDA-approved biodegradable material for anti-cancer drug delivery. ${ }^{17}$ The diameter of PLGA nanospheres was described to be small enough to enable passive tumor targeting through the increased permeation and retention (EPR) effect. ${ }^{18}$ Thus, in this study, a Dox-loaded PLGA nanoparticle (PLGA-Dox) is applied for ovarian cancer treatment. In order to enhance the efficiency of cancer treatment, folic acid (FA) was chosen as the target as it is imperative in DNA synthesis and replication, cell proliferation, survival, and growth. ${ }^{19}$ Noticeably, the FA receptor could be overexpressed in various tumor cells but rarely distributed in normal cells. Also, FA has been recognized to have several advantages such as lower molecular weight, immunogenicity, relatively high stability, and ease of modification. ${ }^{14,15,20}$

In recent years, photothermal therapy (PTT) has been performed as an emerging cancer treatment. Heat generated from the assimilation of optical energy via lightassimilating agents accumulated in the tumor area of postNIR irradiation to kill tumor cells/tissues. ${ }^{21,22}$ Compared with visible light and ultraviolet, NIR laser is a safe light because of the little hurt to healthy and deep penetration. ${ }^{23}$ Despite the efficient tumor ablation of PTT, the application is limited by the possible tumor reoccurrence induced by deficient hyperthermia or asymmetrical heat delivery within tumor areas. ${ }^{24}$ One probable approach to optimize their therapeutic effects is to combine PTT with other types of treatments. It was reported that PTT and chemodrug synergistically enhance the antitumor effect to a great extent. ${ }^{25-29}$ Due to the post-X-ray bombardment, the secondary electrons were distributed to enhance the EPR effect, and consequently, high- $Z$ nanomaterials could optimize the passive tumor targeting. As the atomic number is positively related to the photoelectric interaction, Bi nanoparticles are better than other elements. ${ }^{30}$ Therefore, we considered bismuth sulfide $\left(\mathrm{Bi}_{2} \mathrm{~S}_{3}\right)$ as a PTT agent on account of its outstanding biocompatibility and imaging performance. Furthermore, perfluoropentane (PFP), as a phase change material, would trigger the liquid-gas phase conversion and result in the generation of bubbles post laser irradiation, which could accelerate the tumor cell uptake of Dox. ${ }^{31}$

Overall, with the rapid evolution of diagnostic-image technique, molecular imaging has been recognized as a burgeoning methods for accurate diagnosis guaranteed with high sensitivity and resolution. The establishment of multipurpose probes with the integrated function of cancer-targeting, imaging, and therapeutics are fundamental for accurate oncology. ${ }^{32}$ In contrast to other different imaging methods, X-ray computed tomography (CT) is a mainstream clinical diagnostic strategy with advantages such as high resolution, little depth limitation, and permitting for three-dimensional (3D) reconstruction. ${ }^{33}$ Besides, various internal limitations of CT imaging, especially low soft-tissue contrast and limited approachability, are vital factors that hinder the use of CT for clinical diagnosis. ${ }^{34,35}$ The performance of precision diagnosis can be optimized by the integration of $\mathrm{CT}$ with other imaging strategies that can uncover their preponderances. Comparatively, photoacoustic (PA) imaging, a nonionizing imaging form, transforms photon energy into acoustic pressure waves to obtain pictures, offering comparatively deeper tissue penetration because of the less scattering of acoustic waves than light. ${ }^{8,36}$ Therefore, the design and synthesis of a therapeutic agent have become a major area of interest to effectuate the dual-modal imaging-guided treatment and concurrently maximize the virtue, safety, and precision of cancer treatment.

Herein, we designed the FA-targeted multifunctional NPs loading with Dox, $\mathrm{Bi}_{2} \mathrm{~S}_{3}$ and PFP to achieve an appealing tumor-targeted and dual-modal imaging-guided ovarian cancer combination therapy. In this nano-system, $\mathrm{Bi}_{2} \mathrm{~S}_{3}$ was 
used as the imaging contrast agent and PTT agent, while Dox served as the chemotherapy drug for the treatment of ovarian cancer. PFP as a phase change material was further encapsulated in PLGA. After intravenous administration of FA-Bi ${ }_{2} \mathrm{~S}_{3}$-PFP-Dox-PLGA NPs (described as FBPD NPs), these NPs availably accumulated into the tumor area assisted by active FA targeting after been injected into the mouse. Subsequently, the investigations in vitro and in vivo proved that the FBPD NPs manifest an excellent synergistic effect of PTT and chemotherapy with the guidance of CT and PA imaging, which considerably inhibited the tumor growth in mouse ovarian cancer models (Figure 1).

\section{Materials and Methods Materials}

Dox was purchased from Beijing Bailingwei Technology Co., Ltd. (Mw=579.99, China). $\mathrm{Bi}_{2} \mathrm{~S}_{3}$ modified by oleic acid and PLGA-PEG-FA were obtained from Xi'an Ruixi Biological Technology Co., Ltd. (China). Perfluoropentane (PFP) was obtained from Strem Chemicals, Inc. (America). Cell Counting Kit-8 (CCK-8) was obtained from Dojindo (Japan). The 2-(4-Amidinophenyl)-6-indolecarbamidinedihydrochloride (DAPI) and Trypsin-EDTA solution (0.25\% trypsin) were obtained from Beyotime Technology. Dichloromethane $\left(\mathrm{CHCl}_{2}\right)$ and isopropyl alcohol were obtained from Chongqing Chuan Dong Chemical Co., Ltd.
(China). Poly (vinyl alcohol) (PVA), 1,1'-dioctadecyl $-3,3,3^{\prime}, 3^{\prime}$ - tetramethylindotricarbocyanine iodide (DiR) and Dialysis membrane (10000-12000Da) were obtained from Sigma-Aldrich Chemical Co., Ltd. (MW: 25000, America). McCoy's 5A was obtained from Procell Life Science\&Technology Co., Ltd.

Synthesis of FBPD NPs

PLGA-PEG-FA encapsulating $\mathrm{Bi}_{2} \mathrm{~S}_{3}, \mathrm{PFP}$, and Dox (designed as FBPD NPs) was manufactured by a double emulsion (water/oil/water: W/O/W) method. ${ }^{37}$ First, $10 \mathrm{mg}$ of Dox dissolved in $200 \mu \mathrm{L}$ of double distilled water; Then $400 \mu \mathrm{L}$ of PFP was added to the above solution, followed by a probe sonication under the ice bath $(100 \mathrm{~W}, 1 \mathrm{~min})$. And $200 \mu \mathrm{L}$ of $\mathrm{Bi}_{2} \mathrm{~S}_{3}$ and $50 \mathrm{mg}$ PLGA-PEG-FA were dissolved in $3 \mathrm{~mL}$ of $\mathrm{CHCl}_{2}$, which was mixed with the mixture prepared before. Immediately, the compound was emulsified with an ultrasonic probe to form the initial emulsion ( $100 \mathrm{~W}, 3 \mathrm{~min}$, ice bath). For the second emulsion, $8 \mathrm{~mL}$ of PVA $(\mathrm{w} / \mathrm{v}=4 \%)$ was joined into the initial emulsion and mixed by probe sonication for 2 min (100 W, ice bath). Subsequently, the second emulsion was diluted in $10 \mathrm{~mL}$ of isopropyl alcohol solution $(\mathrm{w} / \mathrm{v}=2 \%)$, and the as-prepared emulsion was stirred at an ice bath for 3 hours to volatilize $\mathrm{CHCl}_{2}$ fully. Finally, the FBPD NPs were collected after the centrifugation $\left(10,000 \mathrm{rpm}, 5 \mathrm{~min}, 4^{\circ} \mathrm{C}\right)$. As for the preparation of FBP

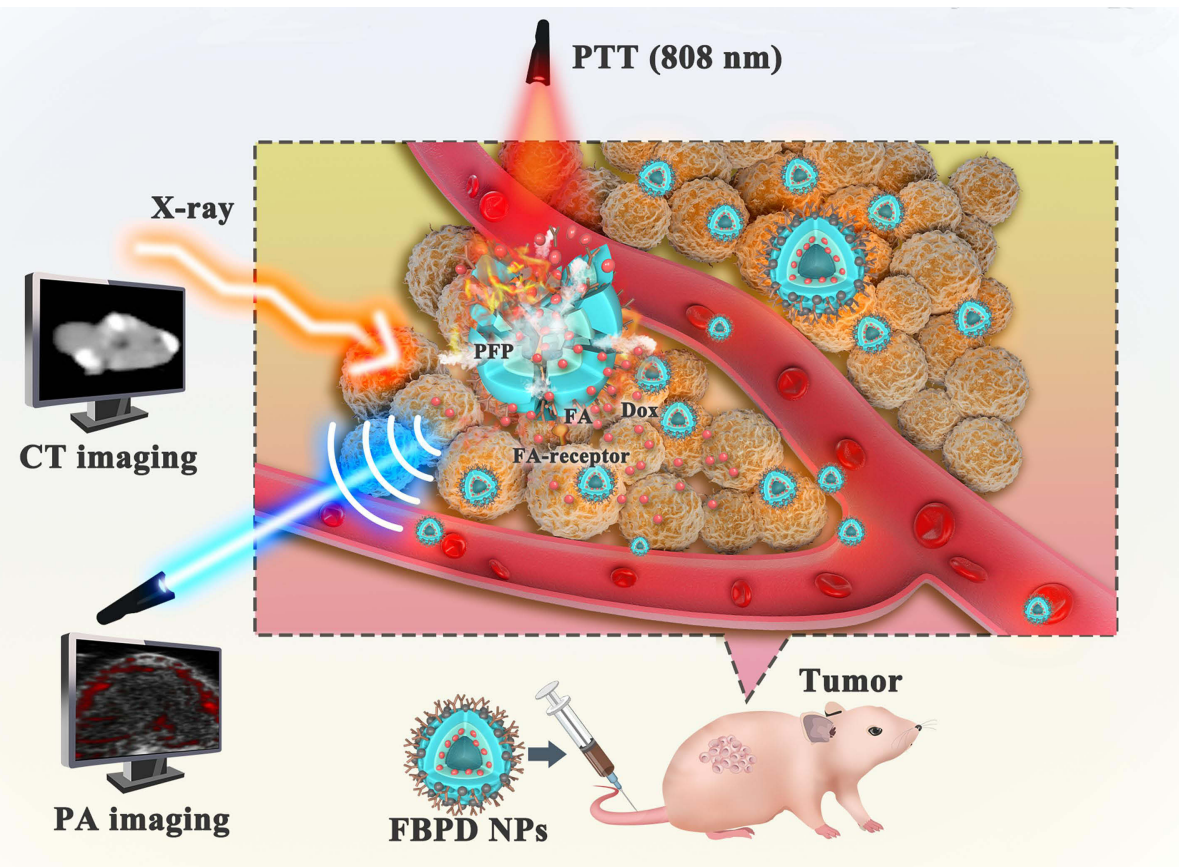

Figure I Schematic illustration of the multipurpose nanoplatform for availably CT/PA imaging-guided combination therapy to tumor cells/tissue. 
NPs or BPD NPs, the difference lies in the removal of FA or Dox. All operations were carried out in dark.

\section{The Characterization of FBPD NPs}

Scanning electron microscopy (SEM, Hitachi S-3400N, Japan) and transmission electron microscopy (TEM, Hitachi H-7600, Japan) were used to characterize the morphology and structure of the FBPD NPs. The zeta potential, average diameter and the polydispersity index (PDI) were determined by Malvern Zetasizer Nano ZS instrument (Malvern Instruments, UK). A UV-vis spectrophotometer (UV-3600, Shimadzu, Japan) was used to assess the UV-vis absorption spectra. The concentration of Dox was determined by a standard curve method using a UV-vis spectrometer. The concentration of $\mathrm{Bi}_{2} \mathrm{~S}_{3}$ was confirmed by inductively coupled plasma mass spectrometry (ICP-MS). The in vivo circulation half-life of the FBPD NPs was also confirmed by inductively coupled plasma mass spectrometry (ICP-MS).

The release of Dox in vitro under laser irradiation was assessed by dialysis. ${ }^{8}$ FBPD NPs were transferred into dialysis bags (MWCO:10,000 Da) that were placed into $50 \mathrm{~mL}$ PBS, with stirring $(150 \mathrm{rpm})$ at $37^{\circ} \mathrm{C}$. FBPD NPs were irradiated with laser $(1.5 \mathrm{~W}, 10 \mathrm{~min})$ or not before stirring. A $1 \mathrm{~mL}$ of dialysate was aliquoted from the flask at different points $(1,2,3,4,6,12,24$, and $48 \mathrm{~h})$, and $1 \mathrm{~mL}$ fresh PBS was added to the flask to remain constant volume. All samples were stored in $-20^{\circ} \mathrm{C}$ before measurement. The absorbance of Dox in the above solutions was measured by UV-Vis spectrophotometer.

\section{Cell Culture}

The human ovarian cancer SKOV-3 cell line was acquired from Procell Life Science \& Technology Co., Ltd. The cells were cultivated in McCoy's 5A medium and maintained in an incubator with $5 \% \mathrm{CO}_{2}$ at $37^{\circ} \mathrm{C}$. When the cells were observed under the microscope to reach the logarithmic growth phase, $0.25 \%$ trypsin-EDTA was employed to harvest the cells for follow-up experiments.

\section{In vitro Cytotoxicity Assay of FBPD NPs}

The SKOV-3 cells were cultivated in 96-well culture plates at a density of $1 \times 10^{4}$ cells/well in McCoy's $5 \mathrm{~A}$ medium at $37{ }^{\circ} \mathrm{C}$ in the condition of $5 \% \mathrm{CO}_{2}$, although the cells adhere to the plates. Then, fresh culture medium containing NPs at the concentration of $2 \mathrm{mg} / \mathrm{mL}$ was used to substitute the above culture medium. Incubation of 3 $\mathrm{h}$ later, the viability of cells was evaluated by the CCK- 8 assay. Partial experimental group were treated by laser at an intensity of $1.5 \mathrm{~W} / \mathrm{cm} 2$ for $5 \mathrm{~min}$. Eight treatment groups were set up in this experiment: the control group (Control), the group subjected to laser only (Laser only), the group operated with FBP NPs (FBP NPs), the group operated with FBP NPs and laser (FBP NPs + laser), the group operated with BPD NPs (BPD NPs), the group operated with BPD NPs and laser (BPD NPs + laser), the group operated with FBPD NPs (FBPD NPs), and the group operated with FBPD NPs and laser (FBPD NPs + laser). Finally, the viability of the cells was assessed by a standard CCK-8 assay.

\section{In vitro Targeting Ability of FBPD NPs}

The cellular uptake effect of FBPD NPs was assessed by confocal laser scanning microscopy (CLSM) and flow cytometry. SKOV-3 cells in logarithmic growth were seeded into CLSM-exclusive culture dishes at a density of $1 \times 10^{5}$ cells/well. After $24 \mathrm{~h}$ of culture, FBPD NPs and BPD NPs were added separately and incubated 0.5, 1, 2, 3, and $4 \mathrm{~h}$, respectively. After co-incubation with nanoemulsions, the cells were washed with PBS, and fixed in paraformaldehyde (4\%) for $15 \mathrm{~min}$. After fixing the cells, SKOV-3 cells were incubated with DAPI fluorescein $(300 \mu \mathrm{L})$ for $15 \mathrm{~min}$. Ultimately, these fluorescence pictures were obtained through CLSM. Furthermore, the quantitative intracellular uptake of FBPD NPs and BPD NPs was analyzed with flow cytometry. In detail, the cells were digested with trypsin $(0.25 \%)$ after incubation with FBPD NPs or BPD NPs, and then suspended in PBS immediately. Finally, the flow cytometry was used to assess the phagocytosis rate.

\section{In vitro and in vivo $C T$ Imaging}

A clinical CT-imaging system (General Electric Company, America) was used to conduct CT-imaging. FBPD NPs dissolved in PBS at different concentrations $(0,1,2,3,4$ and $5 \mathrm{mg} / \mathrm{mL}$ ) were placed in a $2 \mathrm{~mL}$ Eppendorf tube for $\mathrm{CT}$ imaging in vitro. The imaging parameters were set as follows: $170 \mathrm{~mA}, 80 \mathrm{kV}$, and $0.625 \mathrm{~mm}$. The CT-signal intensities of ROI were measured. In the experiment of CT-imaging in vivo, SKOV-3 tumor-bearing mice were i.v. injected with FBPD NPs or BPD NPs solution $(200 \mu \mathrm{L}$, $5 \mathrm{mg} / \mathrm{mL}$ ). Then, CT imaging was conducted at different time points $(0,0.5,1,2,3,4,6$, and $24 \mathrm{~h})$. Average CT signal intensity (SI) of the tumor area of the same slice was measured. 


\section{In vitro and in vivo PA Imaging}

A Vevo LAZR Photoacoustic Imaging System (VisualSonics Inc., Toronto, Canada) was employed to obtain PA images. For in vitro PA imaging, different concentrations of FBPD NPs $(0,1,2,3,4$, and $5 \mathrm{mg} / \mathrm{mL})$ were dissolved in PBS, which was used for PA signal inspection and to assess the linear relation of the PA signal as a function of FBPD NPs concentration. As for PA imaging in vivo, the images were surveyed using SKOV-3 tumorbearing mice, which were configured into two groups (FBPD NPs and BPD NPs) ( $n=3)$. After i.v. injecting 200 $\mu \mathrm{L}(5 \mathrm{mg} / \mathrm{mL})$ BPD NPs or FBPD NPs solution, the homologous PA images were recorded at different time points $(0,0.5,1,2,3,4,6$ and $24 \mathrm{~h})$. Then, the PA signal intensity of each sample was analyzed by Vevo LAZR software.

\section{In vitro Photothermal Imaging and Photo-Induced Phase Change Experiment} In order to verify the PTT effect, the FBPD NPs at different concentrations $(0,1,2,3,4$, and $5 \mathrm{mg} / \mathrm{mL})$ was exposed to an $808 \mathrm{~nm}$ laser $\left(1.5 \mathrm{~W} / \mathrm{cm}^{2}, 5 \mathrm{~min}\right)$, and the infrared thermal images and temperature were reserved by an infrared thermal imaging camera (Fotric 226, China). To further show the photothermal stability of $\mathrm{Bi}_{2} \mathrm{~S}_{3}$, the NPs were irradiated by NIR laser $\left(1.5 \mathrm{~W} / \mathrm{cm}^{2}\right)$ for $5 \mathrm{~min}$ (laser on), then naturally cooled to room temperature (laser off) for five cycles to indicate the cyclical temperature change of FBPD NPs. The infrared thermal images were also recorded. Furthermore, in the experiment of FBPD NPs photo-induced phase changed, the FBPD NPs were placed on an inverted fluorescence microscope post laser irradiation, and the changes before and after the irradiation were recorded.

\section{Animal Model}

All animals (female nude mice with the weight of 16-20 $\mathrm{g}$ and age of 6-8 W) were obtained from Chongqing Tengxin Biotechnology Co., Ltd. All the operation were executed under direction ratified by the Institutional Animal Care and Use Committee of Chongqing Medical University. To construct SKOV-3 tumor-bearing mice models, SKOV-3 cells were suspended into McCoy's 5A medium and then injected subcutaneously to the flank of nude mice.

\section{In vivo Targeting Ability of FBPD NPs}

To study the distribution of FBPD NPs in vivo, six SKOV3 tumor-bearing mice were divided into two groups: FBPD NPs group and BPD NPs group $(n=3)$. Firstly, all the mice were injected with FBPD NPs or BPD NPs $(200 \mu \mathrm{L}, 5 \mathrm{mg} /$ $\mathrm{mL}$ ) intravenously; then, the fluorescence pictures were collected at certain moments $(0,0.5,1,2,3,4,6$, and $24 \mathrm{~h})$ after injecting NPs by a fluorescence system (Fx7 Ir Spectra, Vilber Lourmat, France). The excitation and emission wavelengths of $748 \mathrm{~nm}$ and $780 \mathrm{~nm}$.

\section{In vivo Tumor Growth Inhibition of FBPD NPs}

To further evaluate the in vivo chemotherapy/photothermal efficacy of FBPD NPs, eight groups were established as the in vivo experiment $(\mathrm{n}=5)$. When the tumor volume reached approximately $50-80 \mathrm{~mm}^{3}$, the SKOV-3 tumorbearing mice were subjected to different treatments as follows: a. the group was i.v. injected with saline solution $(200 \mu \mathrm{L})$ (control group); b. the group was treated with laser only $\left(1.5 \mathrm{~W} / \mathrm{cm}^{2}, 5 \mathrm{~min}\right)$ (Laser only); c. the group was i.v. injected with FBP NPs $(200 \mu \mathrm{L}, 5 \mathrm{mg} / \mathrm{mL})(\mathrm{FBP}$ NPs); d. the group was i.v. injected with FBP NPs (200 $\mu \mathrm{L}, 5 \mathrm{mg} / \mathrm{mL})$ followed by $808 \mathrm{~nm}$ laser $\left(1.5 \mathrm{~W} / \mathrm{cm}^{2}, 5\right.$ min) $3 \mathrm{~h}$ later (FBP NPs + laser); e. the group was i.v. injected with BPD NPs $(200 \mu \mathrm{L})$ at the concentration of $5 \mathrm{mg} / \mathrm{mL}$ (BPD NPs). f. the group was i.v. injected with BPD NPs $(200 \mu \mathrm{L}, 5 \mathrm{mg} / \mathrm{mL})$ followed by $808 \mathrm{~nm}$ laser $\left(1.5 \mathrm{~W} / \mathrm{cm}^{2}, 5 \mathrm{~min}\right) 3 \mathrm{~h}$ later (BPD NPs + laser); g. the group was i.v. injected with FBPD NPs $(200 \mu \mathrm{L}, 5 \mathrm{mg} /$ $\mathrm{mL}$ ) (FBPD NPs); h. the group was i.v. injected with FBPD NPs $(200 \mu \mathrm{L}, 5 \mathrm{mg} / \mathrm{mL})$ followed by $808 \mathrm{~nm}$ laser $\left(1.5 \mathrm{~W} / \mathrm{cm}^{2}, 5 \mathrm{~min}\right) 3 \mathrm{~h}$ later (FBPD NPs + laser). After various treatments, the changes in tumor-volume and the weight of each mouse were recorded every two days. The relative tumor volumes $\mathrm{V} / \mathrm{V}_{0}\left(\mathrm{~V}_{0}\right.$ : the initial tumor volume before the treatment) were used to express the tumor-volume changes. The tumor tissues and the main organs (heart, liver, spleen, lung, and kidney) of one mouse of each group were collected after treatment, and then steeped in a $4 \%$ paraformaldehyde solution. Ultimately, the primary organs were stained with H\&E, and the tumor were stained with H\&E, TUNEL and PCNA for histopathological analysis. 


\section{Biosafety Assay of FBPD NPs}

Twenty-five Balb/c mice were i.v. injected with FBPD NPs $(5 \mathrm{mg} / \mathrm{mL}, 200 \mu \mathrm{L})$ and were divided into five groups (1, $3,7,14$, and 28 days after the injection). Five Balb/c mice were injected with saline as the control group. Blood samples were used for the blood index including routine blood and biochemistry, and the major organs were used for $\mathrm{H} \& \mathrm{E}$ staining.

\section{Statistical Analysis}

All statistical analyses were executed with SPSS 20.0 software. Data were offered as mean \pm standard deviation. The significance of the data is analyzed according to a Student's $t$-test: $* \mathrm{P}<0.01$.

\section{Results and Discussion}

Design, Synthesis, and Characterization of FBPD NPs

The multipurpose FBPD NPs were synthesized following the strategy as shown in Figure 2A. The double-emulsion approach to synthesize FBPD NPs has been reported by our group before has been skillfully operated. ${ }^{38}$ A series of characterizations have been conducted on the assynthesized NPs. The TEM images (Figure 2C) showed that the FBPD NPs had a well-defined spherical shape, and the SEM image (Figure 2B) revealed that homogeneous size distribution of FBPD NPs. The mean hydrodynamic diameter of the FBPD NPs was $236.2 \pm 33.17 \mathrm{~nm}$ (Figure 2D). The PDI values of the FBPD NPs were

A

Dox

$\mathrm{Bi}_{2} \mathrm{~S}_{3}$

Folic acid

Liquid PFP

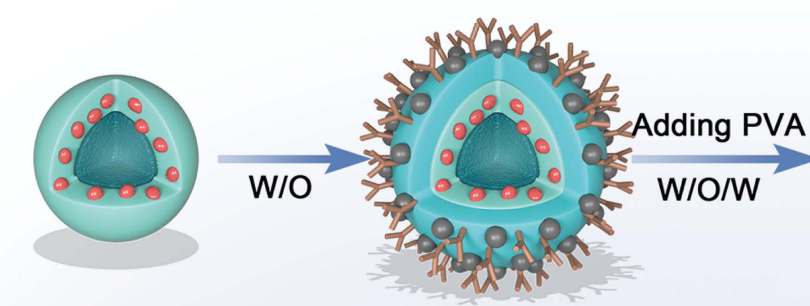

B

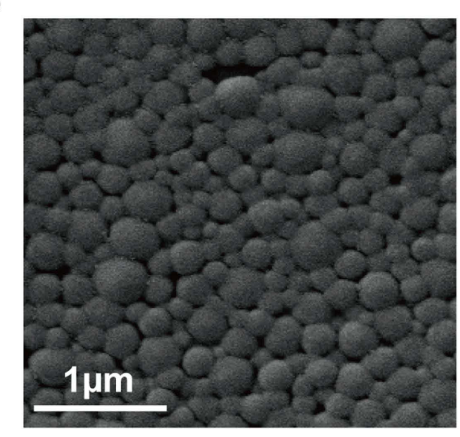

E

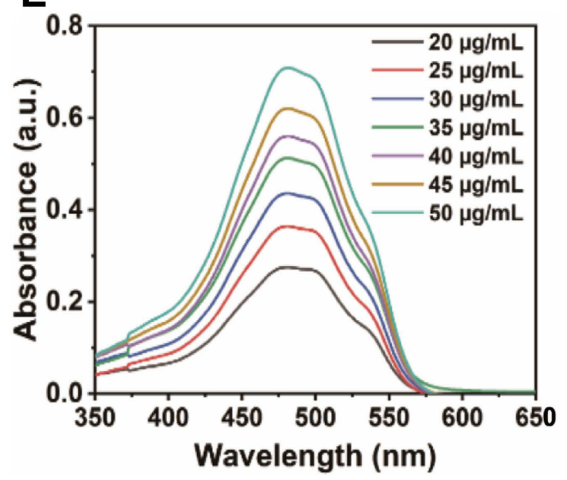

C

$\mathbf{F}$

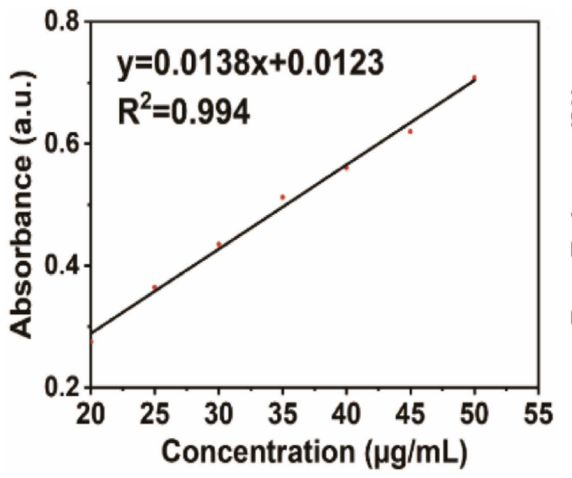

Adding isopropyl alcohol

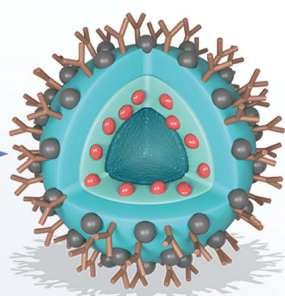

D

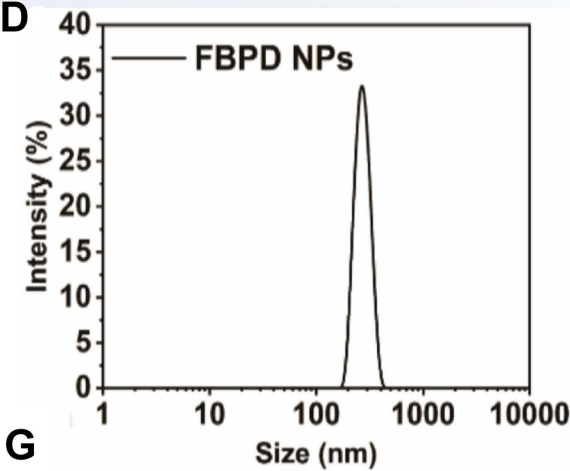

Figure 2 Morphology and characterization. (A) Schematic diagram for the fabrication of FBPD NPs. (B) SEM and (C) TEM of FBPD NPs. (D) Size distribution of FBPD NPs. (E) UV-spectra of Dox dispersed in saline in different concentrations $(20,25,30,35,40,45,50 \mu \mathrm{g} / \mathrm{mL})$ and $(\mathbf{F})$ corresponding relationship between concentration of Dox and absorbance. (G) In vitro release of DOX in FBPD NPs with or without laser irradiation. 
0.147 , demonstrating a narrow size distribution of these fabricated nanoparticles. The size range indicated that it could readily transport in the blood vessel and accumulate into tumor tissue via the representative enhanced permeability and retention (EPR) effect. ${ }^{37}$ In addition, the zeta potential of FBPD NPs was $(-7.99 \pm 6.66) \mathrm{mV}$, a favorable negative potential. The negative zeta potential may facilitate the nanodroplets to repel each other and prevent aggregation in vivo. Furthermore, as has been proved recently, different drug delivery systems with a negatively charged surface are generally less toxic compared to the positively charged ones. ${ }^{39} \mathrm{UV}$-spectra of Dox dispersed in saline at different concentrations $(0,25,30$, $35,40,45$ and $50 \mu \mathrm{g} / \mathrm{mL}$ ) and the corresponding relationship between concentrations of Dox and absorbance (Figure 2E and F). The EE and DL of Dox were calculated by UV spectrophotometry, the EE and DL of $\mathrm{Bi}_{2} \mathrm{~S}_{3}$ were calculated by ICP-MS. The EE was $(87.48 \pm 0.19 \%)$ for $\mathrm{Bi}_{2} \mathrm{~S}_{3}$ and $(25.21 \pm 3.05 \%)$ for DOX, respectively. The DL was $(3.38 \pm 0.07 \%)$ for $\mathrm{Bi}_{2} \mathrm{~S}_{3}$ and $(4.07 \pm 0.49 \%)$ for DOX, respectively. The result displayed that FBPD NPs could carry oil-solute and soluble drugs. While these FBPD NPs could act as photothermal conversion agents for therapeutic tumor ablation, due to its excellent imaging performance, these FBPD NPs could be potentially used as contrast agents for CT and PA simultaneously, offering guidance for subsequent photothermal ablation and evaluating the potential of treatment results. To study the systemic circulation time of FBPD NPs, $200 \mu \mathrm{L}$ of $5 \mathrm{mg} / \mathrm{mL}$ FBPD NPs were injected into three mice. At various time points after the injection, $10 \mu \mathrm{L}$ of blood was collected from the eye socket of the mice for ICP-MS analysis. As shown in Figure S1, the elimination half-life was calculated as $3.77 \mathrm{~h}$ for the FBPD NPs and the concentration of FBPD NPs in the body was only $3.5 \mu \mathrm{g} / \mathrm{mL}$ at $24 \mathrm{~h}$, demonstrating that the FBPD NPs are excellent in vivo clearance and have the potential to be a good contrasting agent.

Different releasing behaviors were observed for Dox for FBPD NPs when in the presence and absence of laser irradiation (Figure 2G). The percentage of Dox released almost reached $46.2 \%$ at $4 \mathrm{~h}$ after laser irradiation, while the percentage of Dox released reached $49.7 \%$ at $48 \mathrm{~h}$ in the control group. This is probably due to the addition of phase-changing PFP into the FBPD NPs. The laser irradiation triggered the liquid-gas phase conversion and subsequently induced a faster release of Dox.

\section{In vitro Cytotoxicity Assay of FBPD NPs}

The cytotoxicity against SKOV-3 cells was assessed by the CCK-8 protocol. As shown in Figure 3, the cell viabilities of the FBPD NPs + Laser group was only 9.6\%, demonstrating that the combination of PTT and drug-loaded nanoparticles with active targeting ability could significantly improve the killing efficiency. In addition, the cell viabilities of FBP NPs + laser group was $14.1 \%$, ranking second to FBPD NPs + laser group. In contrast, the FBP NPs group exhibited $99 \%$ cell survival rate, demonstrating almost no killing effect on ovarian cancer cells. This result indicated that PTT is an efficient way for partial tumor remedy utilizing light-responsive nanoparticles and light source. Normally, NIR light-responsive nanoparticles including $\mathrm{Bi}_{2} \mathrm{~S}_{3}$ are irradiated by an NIR laser producing heat, which triggers cancer cell death. However, in the absence of laser irradiation and chemotherapy drugs, FBP NPS did not cause damage to cells, indicating that $\mathrm{Bi}_{2} \mathrm{~S}_{3}$ has a good biosecurity. Furthermore, the cell survival rate of BPD NPs group showed significantly higher than that of FBPD NPs group, approximately 1.9-fold than the latter group, demonstrating that FA could effectively increase the active targeting ability of nanoparticles and facilitate the efficient delivery of drugs into tumor cells, thus enhancing the therapeutic effect of tumor.

\section{In vitro Targeting Ability of FBPD NPs}

Efficient cellular uptake of NPs the requirement to guarantee their therapeutic efficacy. Folate has been shown to increase the cellular uptake of nanocarrier systems by targeting the folate receptor. ${ }^{40}$ Confocal microscopy and flow cytometry were used to investigate the intracellular uptake of FBPD NPs. As shown in CLSM images, NPs contained Dox present red fluorescence, and DAPI-labeled SKOV-3 cells exhibit blue fluorescence (Figure 4A). As expected, in the treatment group, numerous FBPD NPs with red fluorescence were internalized within the cells after $3 \mathrm{~h}$ of co-incubation. On the other hand, only a few BPD NPs assembled within the cells in the control group. Flow detection shows the same trend (Figure 4B). The intercellular uptake of FBPD NPs and BPD NPs was in a time-dependent manner within $3 \mathrm{~h}$ of incubation, indicating that both nanoparticles could enter the cells. But with the guidance of FA, intercellular uptake efficacy of FBPD NPs was much higher than that of BPD NPs. Especially after $3 \mathrm{~h}$ of incubation, the uptake of FBPD NPs by the 


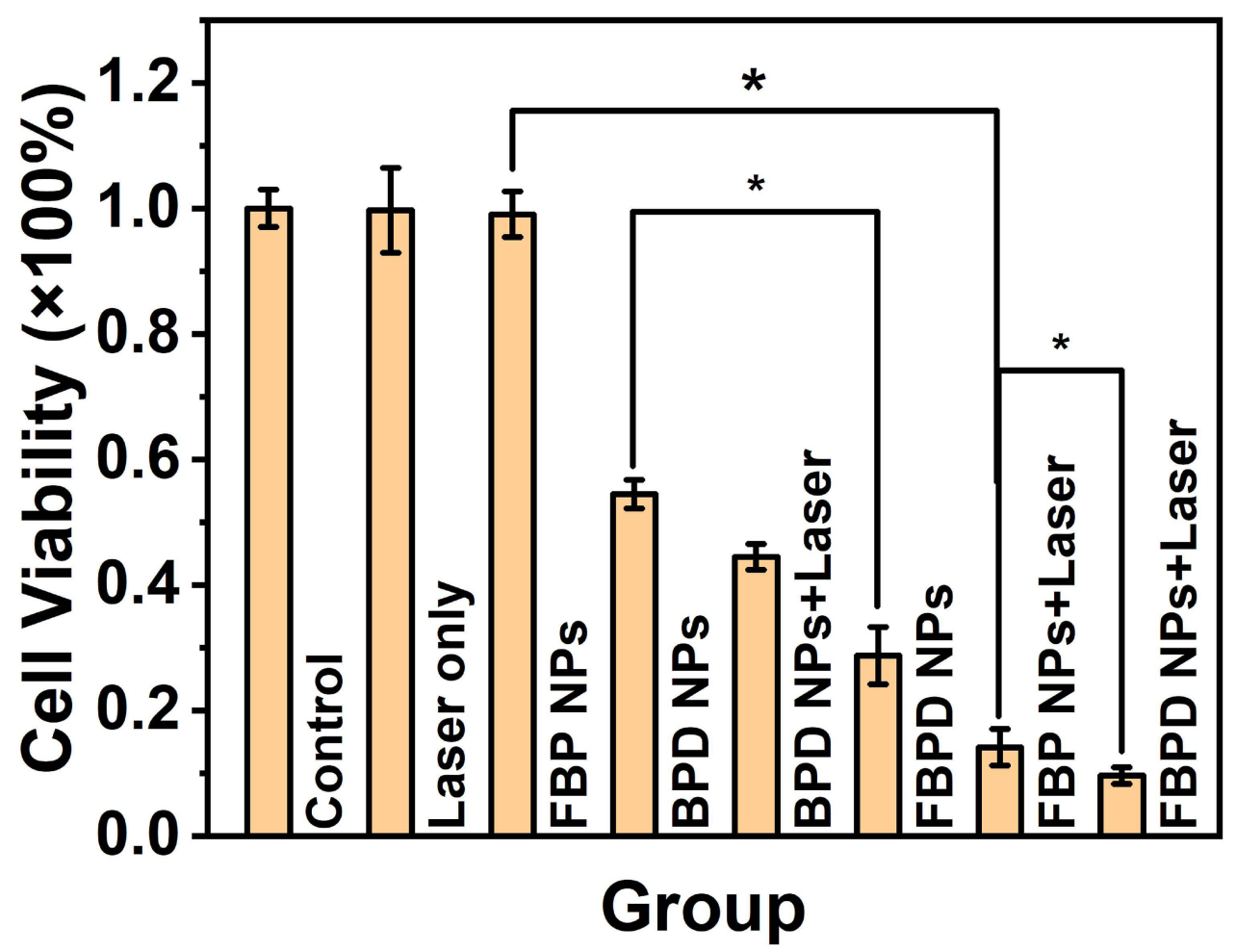

Figure 3 Relative cell viability of SKOV-3 cells after different treatments $\left(n=3, *_{p}<0.0\right.$ I).

SKOV-3 cells reached the peak. The results revealed that FBPD NPs could deliver Dox more efficiently into FAreceptor overexpressed cells.

\section{In vitro and in vivo $C T$ Imaging}

Based on deep tissue penetration and 3D structure imaging advantages, CT imaging is one of the most helpful diagnostic methods. ${ }^{41,42}$ The high atomic number of bismuth has strong X-ray absorption ability, which can significantly improve the contrast of $\mathrm{CT}$ imaging. Furthermore, binanoparticles have the advantages of low toxicity, small particle size, long blood circulation time, and surface modification. ${ }^{43}$ Therefore, the experiment investigated the CT contrast imaging both in vitro and in vivo of FBPD NPs. When in vitro, it has been found that as the concentration of FBPD NPs increased, the CT signal value also increased and showed a good linear relationship (Figure 5A). The CT imaging property in vivo was further evaluated on SKOV-3 tumor-bearing mice. As shown in Figure 5C, after i.v. injection of FBPD NPs or BPD NPs, the group of FBPD NPs showed a distinct bright effect within the tumor area. The CT signal intensities of the tumor regions were measured with Pacs software, and it showed that the CT signal value peaked at $3 \mathrm{~h}$ (Figure 5B). Furthermore, the pseudo-colored images were also recorded, it clearly indicated the increase of CT imaging. This result demonstrated that the targeted nanoparticles coated with $\mathrm{Bi}_{2} \mathrm{~S}_{3}$ could effectively gather in the tumor area and efficiently enhance the effect of CT imaging.

\section{In vitro and in vivo PA Imaging}

To evaluate the capability of FBPD NPs as a PA probe, PA images were obtained using FBPD NPs as the contrast agent both in vivo and in vitro. Compared with other imaging methods, PA imaging has very high sensitivity. ${ }^{24}$ $\mathrm{Bi}_{2} \mathrm{~S}_{3}$ has been used as a PA contrast agent because of its strong absorbance in the NIR region. ${ }^{41}$ While applied in vitro, PA imaging performance of FBPD NPs dispersed in PBS at different concentrations was recorded (Figure 6A). Furthermore, the PA signal intensities increased linearly with the concentration of NPs increased from 1 to $5 \mathrm{mg} / \mathrm{mL}$, suggesting that FBPD NPs could efficiently transform the absorbed energy into heat and trigger ultrasonic wave subsequently. During the in vivo assessment, PA imaging of SKOV-3 tumor-bearing mice was conducted at different time points after i.v. injection of FBPD NPs or BPD NPs (Figure 6B), and the relevant quantitative analyses of $\mathrm{PA}$ intensities were executed (Figure 6C). Before i.v. injection, the tumor showed only a weak PA signal. The PA signal reached the maximum 


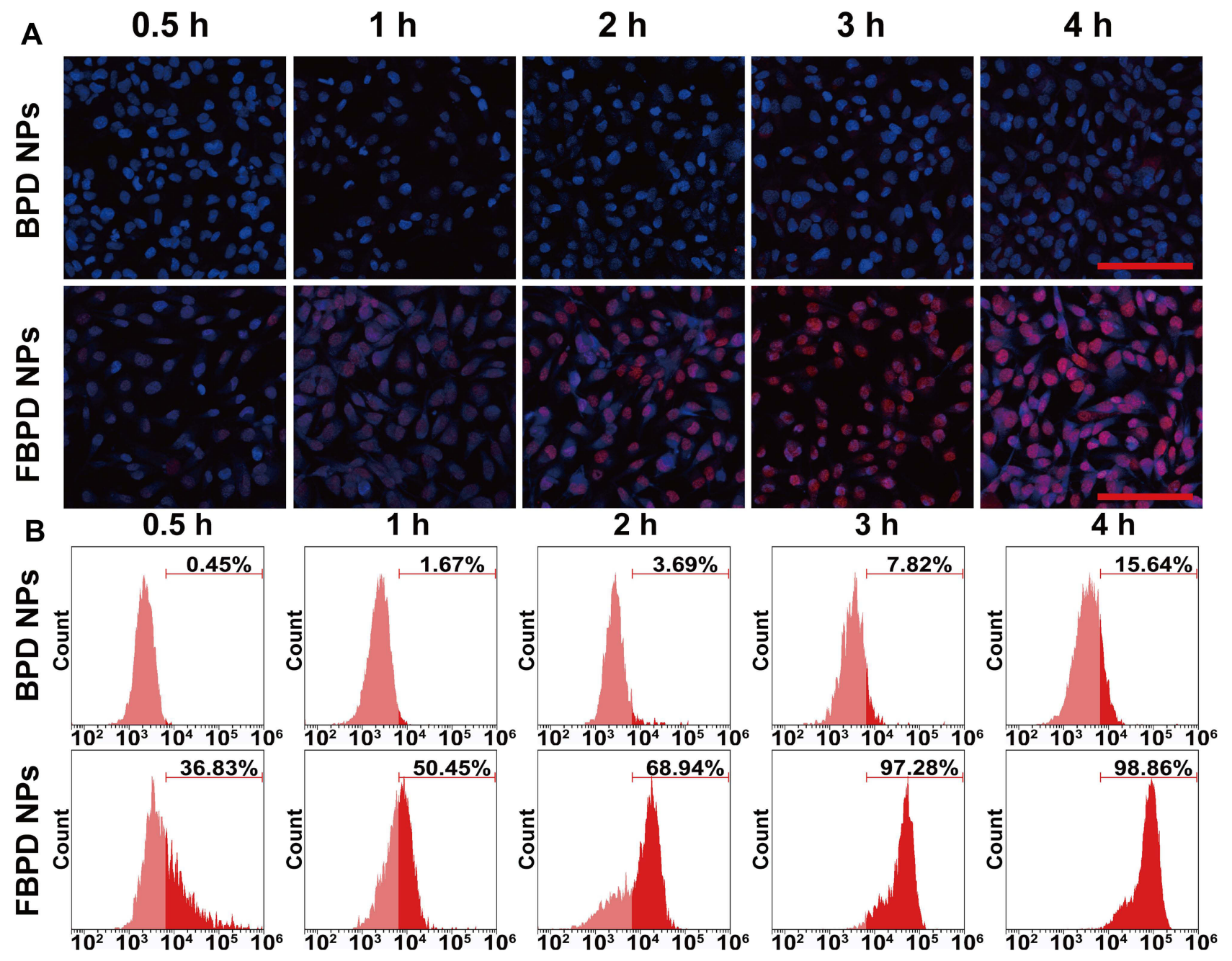

Figure 4 In vitro targeting ability. (A) Intracellular uptake of BPD NPs and FBPD NPs as observed by CLSM after various intervals of incubation. The scale bars are I00 $\mu$ m. (B) Flow cytometry analysis of intracellular uptake of BPD NPs and FBPD NPs.

approximately $3 \mathrm{~h}$ after injection with FBPD NPs. The reason could be the actively tumor-targeting induced by FA modification tumor cells at $3 \mathrm{~h}$, and specifically bind to the folate-receptor on the tumor surface resulting in a high degree of accumulation of FBPD NPs in the tumor. In contrast to the FBPD NPs injection, few PA signal was discovered after BPD NPs injection. These results demonstrated that FBPD NPs could be served as a excellent contrast agent for PA imaging, which achieves diagnosticimaging guidance and monitoring during tumor therapy.

\section{Photothermal and Photo-Induced Phase Change Performance of FBPD NPs}

To assess the photothermal performance of FBPD NPs, the temperature changes of FBPD NPs aqueous solution were recorded by an infrared thermal imaging camera after the
NIR irradiation $(808 \mathrm{~nm})$. After laser irradiation at $1.5 \mathrm{~W} /$ $\mathrm{cm}^{2}$ for $5 \mathrm{~min}$, FBPD NPs showed a rapid temperature increase at all concentrations. FPBD NPs revealed a rapid temperature increase of more than $50^{\circ} \mathrm{C}$ at the maximum concentration of $5 \mathrm{mg} / \mathrm{mL}$ after the laser irradiation. Comparatively, the PBS did not show any obvious temperature rise as the given conditions (Figure 7A), indicating that the existence of FBPD NPs can effectively and quickly transform NIR light into thermal energy. During the laser on-off cycles, the maximum temperature of FBPD NPs did not drop significantly after the cycle, indicated that it has good thermal stability (Figure 7B).

In the experiment of photo-induced phase change, before laser irradiation, FBPD NPs did not change significantly under the optical microscope (Figure 7C); After exposed to laser irradiation, FBPD NPs undergone a liquid-gas phase change, and the volume of FBPD NPs 


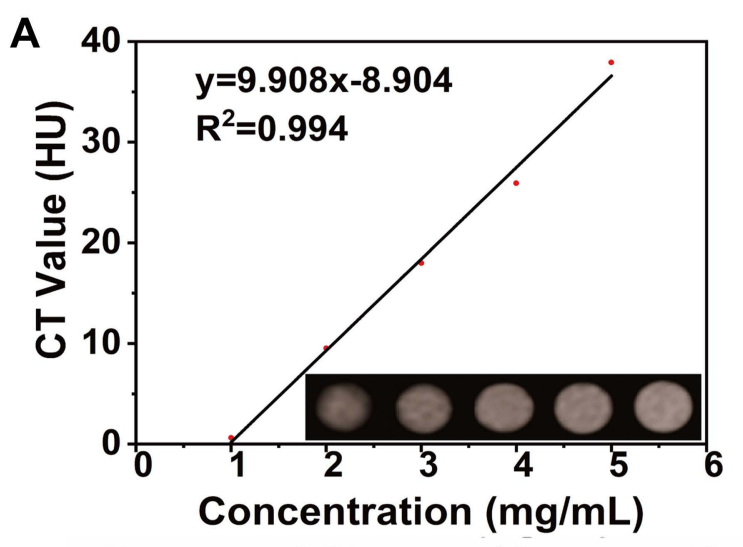

B Pre

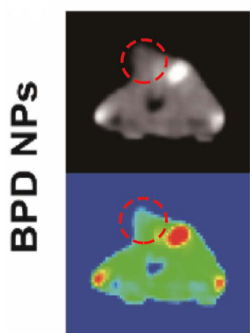

$0.5 \mathrm{~h}$
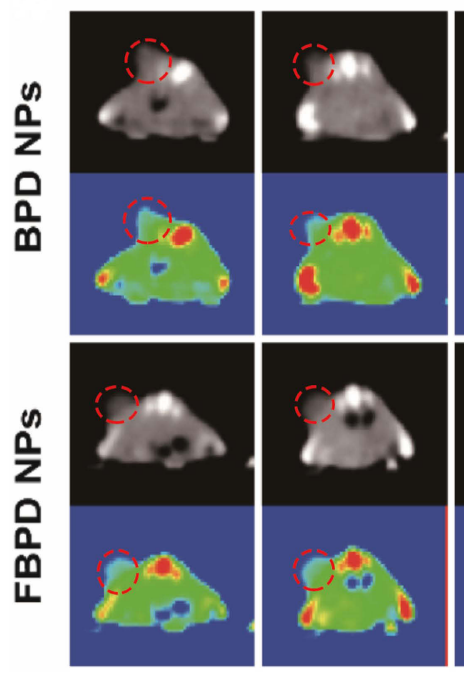

$1 \mathrm{~h}$
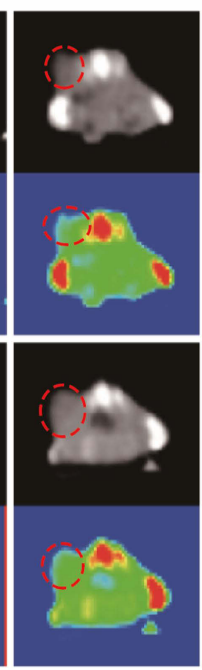

$2 \mathrm{~h}$
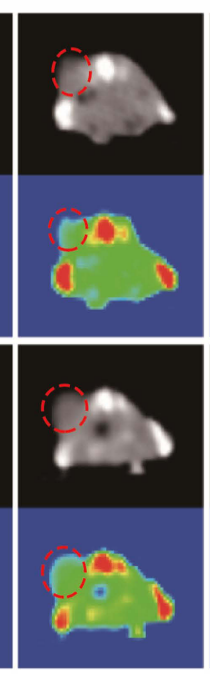

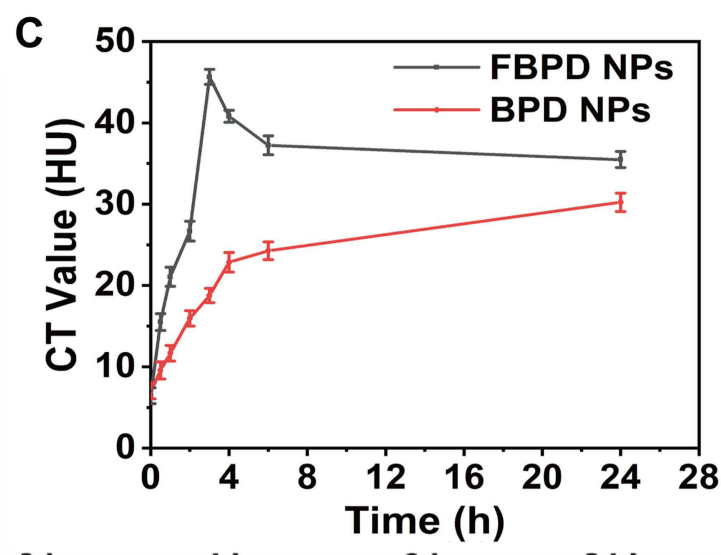

$3 \mathrm{~h}$

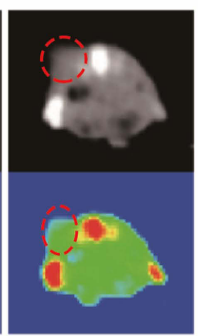

$4 \mathrm{~h}$

$6 \mathrm{~h}$
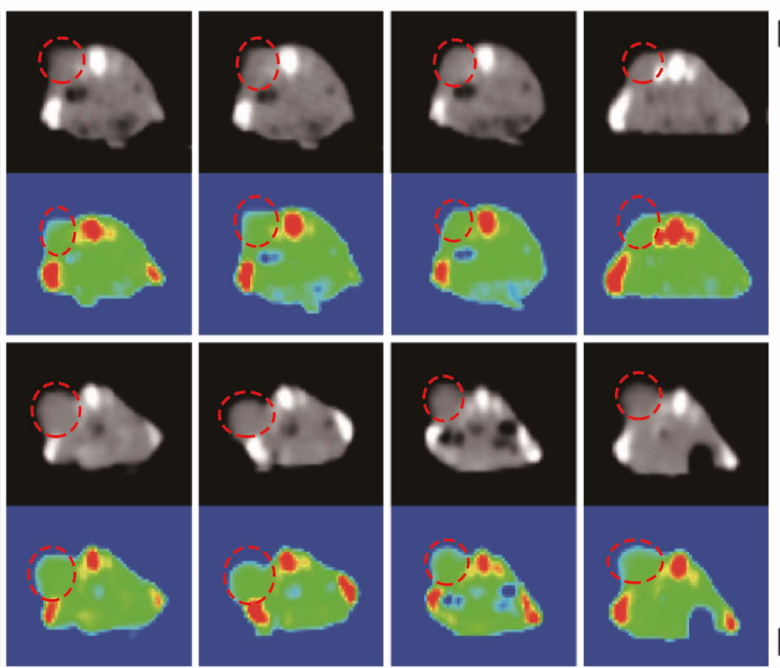

High

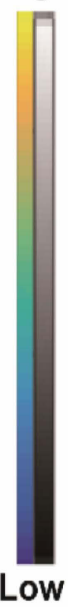

Figure $\mathbf{5}$ In Vitro and In Vivo CT Imaging. (A) In vitro CT contrast images and CT values of FBPD NPs at different concentrations. (B) In vivo CT images of tumors on SKOV-3 tumor-bearing mice after i.v. injection of BPD NPs or FBPD NPs as recorded at different time points (0, 0.5, I, 2, 3, 4, 6, and 24 h). The top row shows black and white images, and bottom row represents the pseudo-colored images. (C) Changes of CT-signal intensities within tumor regions at corresponding time points.

increases significantly (Figure 7D). This result indicated that the NPs could easily transform into gas while heating to physiological temperature due to the lower boiling point of PFP. ${ }^{21}$ The PFP had eventuated a phase change after being heated, then resulted in increase in the size of the FBPD NPs, which was subsequently more helpful for the release of drugs and the thermal ablation effect in the tumor area.

\section{In vivo Targeting Ability of FBPD NPs}

To further verify the targeting efficiency in vivo, the SKOV-3 tumor-bearing mice model was created, and fluorescence pictures were executed at certain time points after the intravenous administration with DiR-labeled FBPD NPs or BPD NPs. After injection of DiR-stained FBPD NPs, strong fluorescence signals were observed in the tumor area, while only weak signals were found in tumors in mice treated with DiR-stained BPD NPs (Figure 8A). According to Figure 8B, the fluorescence intensities with tumor regions reached a peak at 3 hours after FBPD NPs administration. Furthermore, in order to determine the distribution of NPs in the body, main organs and tumors were collected 24 hours postinjection for excised fluorescence imaging (Figure 8C), and the relevant fluorescence intensities were quantified (Figure 8D). As shown, strong fluorescence signals highlighted the liver and spleen tissues in both two groups because of the phagocytosis of the reticuloendothelial system (RES). Moreover, the fluorescence intensity of the tumor in FBPD NPs group showed significantly stronger than that in BPD NPs group, approximately 1.96-fold than the latter group. These results revealed that FBPD NPs show an effectively selective accumulation in tumor. 
A

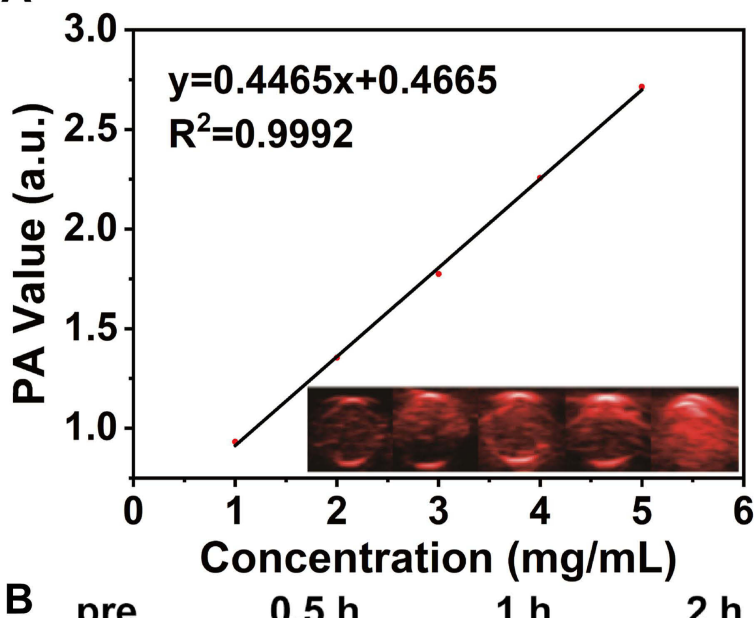

C

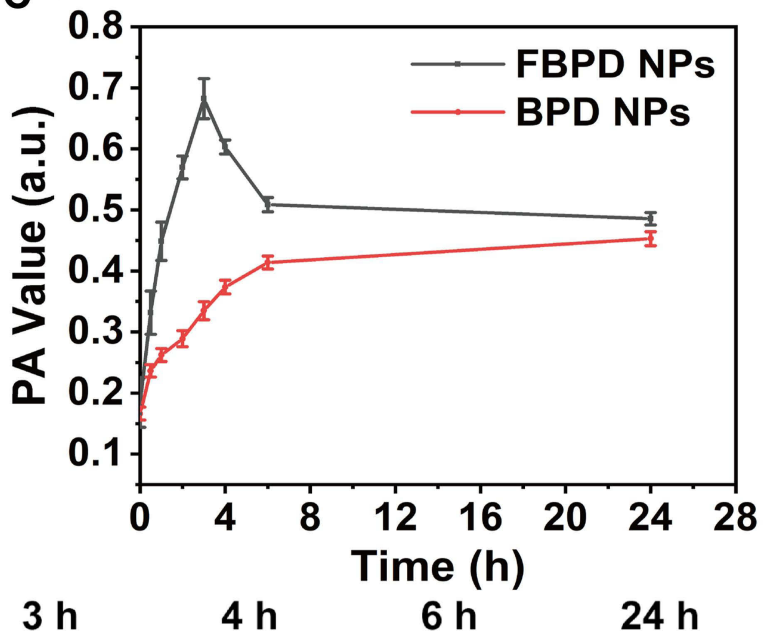

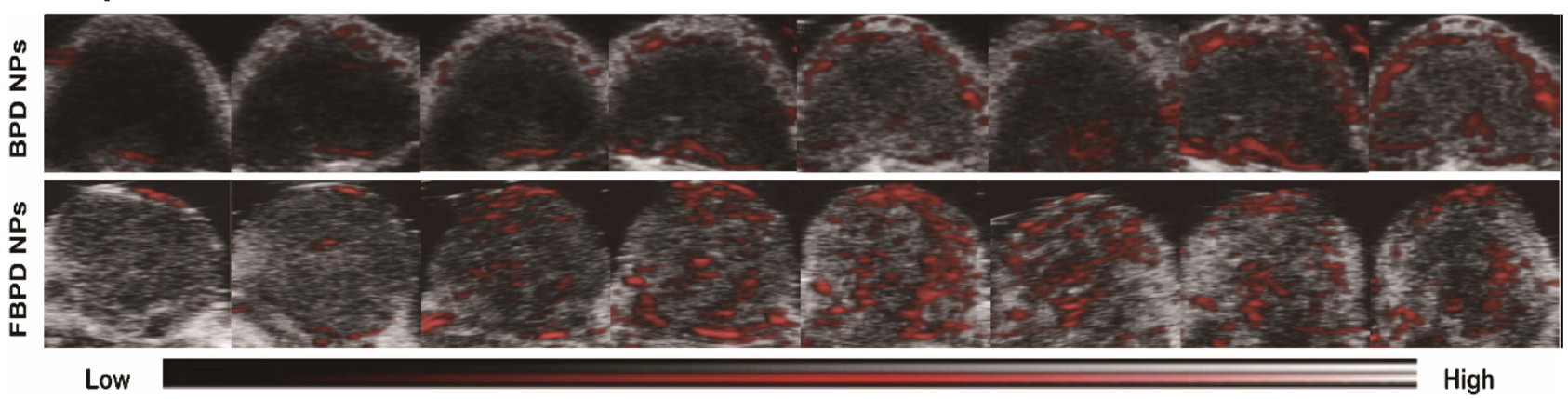

Figure 6 In vitro and in vivo PA imaging. (A) In vitro PA contrast images and PA values of FBPD NPs at different concentrations. (B) PA images of tumor regions in SKOV-3 bearing-mice after i.v. injections of BPD NPs or FBPD NPs at varied time intervals $(0,0.5,1,2,3,4,6$, and 24 h). (C) PA signal at tumor regions in SKOV-3 tumor-bearing mice.

\section{In vivo Cytotoxicity Assay of FBPD NPs}

The combination of PTT and chemotherapy, an efficient therapy for tumors, causes less damage to surrounding normal tissues. According to cytotoxicity assay in vivo, eight groups (control, laser only, FBP NPs group, FBP NPs + laser group, BPD NPs group, BPD NPs + laser group, FBPD NPs group, and FBPD NPs + laser group) were performed to evaluate the therapeutic efficacy. After conducting different treatments, the inhibitory effect on SKOV-3 tumor growth is shown in Figure 9B. The volumes of tumor and the weights of mice were measured every two days (Figure 9C and D); meanwhile, photographs of these mice were taken every two days for fourteen days after the therapy (Figure 9A). The effect of chemotherapy could be fully verified by comparing the FBPD NPs + Laser group and FBP NPs + Laser group.
It is known that the tumor cells are difficult to eliminate completely by only relying on PTT because the heat of NIR light is reduced with the increase of tissues depth because of light scattering and absorption. Besides, tumors treated with FBP NPs showed no therapeutic effect. In contrast, in the FBP NPS + Laser group, tumor tissues were effectively ablated. These results demonstrated that upon laser irradiation, FBP NPs-mediated hyperthermia could ablate tumor cells/tissues. Generally, $\mathrm{Bi}_{2} \mathrm{~S}_{3}$-based NIR light-responsive nanoparticles generate heat irradiated by an NIR light, resulting in cancer cell death. However, in the absence of laser irradiation and chemotherapy drugs, FBP NPS did not cause damage to tissues, indicating the biosafety of $\mathrm{Bi}_{2} \mathrm{~S}_{3}$. At the same time, the weight of body is also an important factor to assess the nanoparticle toxicity. In vivo cytotoxicity assay, the body weight of the eight 


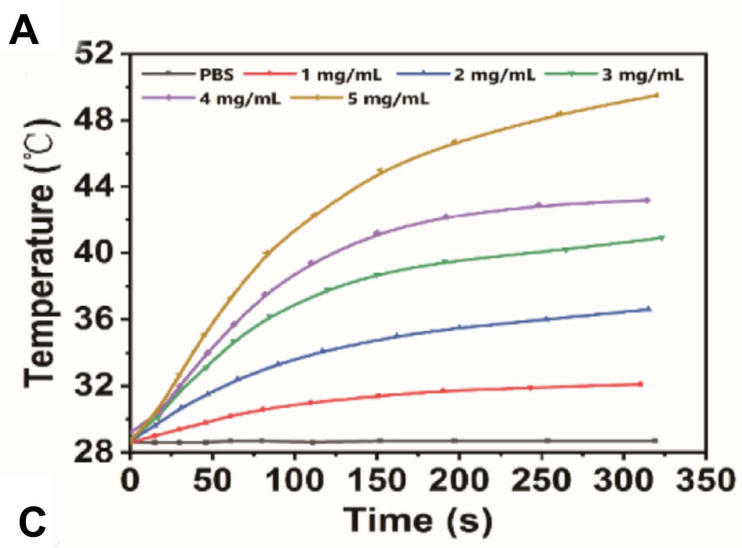

B
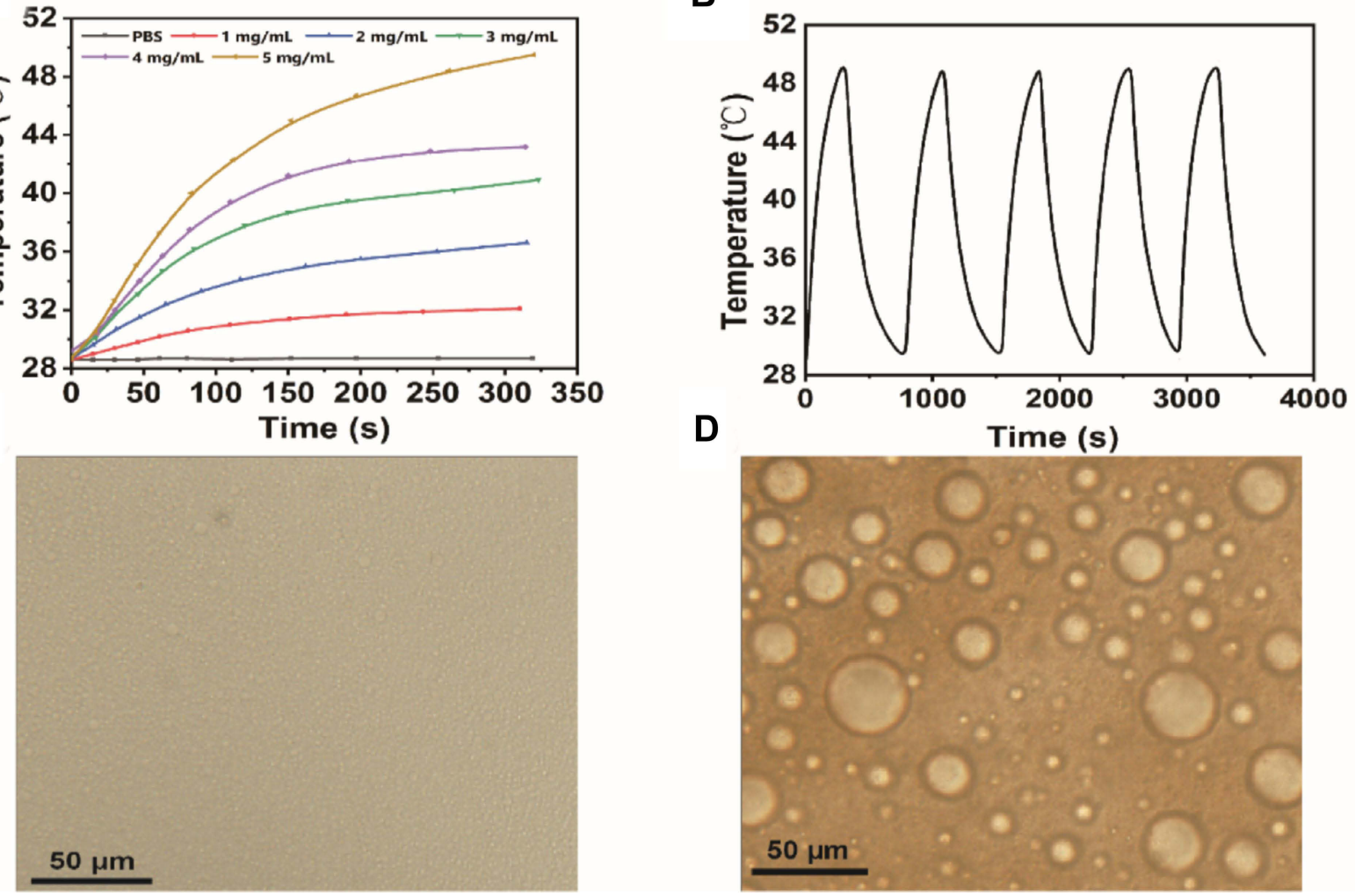

Figure 7 Photothermal and Photo-induced Phase Change performance. (A) Plot of temperature change $(\Delta T)$ of PBS and FBPD NPs at different concentrations (0, I, 2, 3, 4, and $5 \mathrm{mg} / \mathrm{mL}$ ) as a function of irradiation duration using an $808 \mathrm{~nm}$ laser $\left(1.50 \mathrm{~W} / \mathrm{cm}^{2}\right)$. (B) Cycled heating profiles of FBPD NPs (concentration: $100 \mu \mathrm{L}$, $\left.5 \mathrm{mg} / \mathrm{mL}\right)$ irradiated by an $808 \mathrm{~nm}$ laser $\left(1.50 \mathrm{~W} / \mathrm{cm}^{2}\right)$ for five laser on/off cycles. (C) The imaging of FBPD NPs under the optical microscope before an $808 \mathrm{~nm}$ laser (I.50 W/cm $\left.{ }^{2}\right)$. (D) The imaging of FBPD NPs under the optical microscope after an $808 \mathrm{~nm}$ laser $\left(1.50 \mathrm{~W} / \mathrm{cm}^{2}\right)$.

groups did not decrease during treatment, suggesting ignorable side effect of these synthetic nanoparticles. The result demonstrated that FA-loaded NPs could deliver Dox and $\mathrm{Bi}_{2} \mathrm{~S}_{3}$ to the tumor area and specifically kill the tumor. Furthermore, it had been reported that hyperthermia at $41^{\circ} \mathrm{C}-45^{\circ} \mathrm{C}$ would incur a synergistic effect together with traditional therapeutic approaches such as chemotherapy in tumor treatment. ${ }^{44}$ Moreover, the NIR laser which transforms optical energy to heat could penetrate tissue deeply owning to its feebleness absorbance by skin and tissue. ${ }^{45}$ Our experimental results presented consistency with reported mechanisms.

Hematoxylin-eosin (H\&E) staining, proliferating cell nuclear antigen (PCNA) staining, and TdT-mediated dUTP Nick-End Labeling (TUNEL) staining on tumor slices better affirmed the synergistic PTT/chemotherapy effect (Figure 10A). As shown in the H\&E-staining tumor sections, there were much more deformed nucleus in FBPD NPs + Laser group than tumor sections in other groups, prompting serious necrosis of tumor cells. The result of TUNEL and PCNA staining followed the parallel tendency. Representative apoptosis cells were prompted by brown nucleus in the TUNEL staining. PCNA staining on tumor slices showed the in vivo proliferative abilities of eight groups where proliferative tumor cells were stained into brown. The TUNEL and PCNA staining indicated that synergistic PTT + chemotherapy possessed the highest efficacy than other groups. In addition, heart, liver, spleen, lung, and kidney of mice were conducted H\&E staining after the treatments to survey the relevant pathological toxicity. Insignificant negative effect was discovered during the 

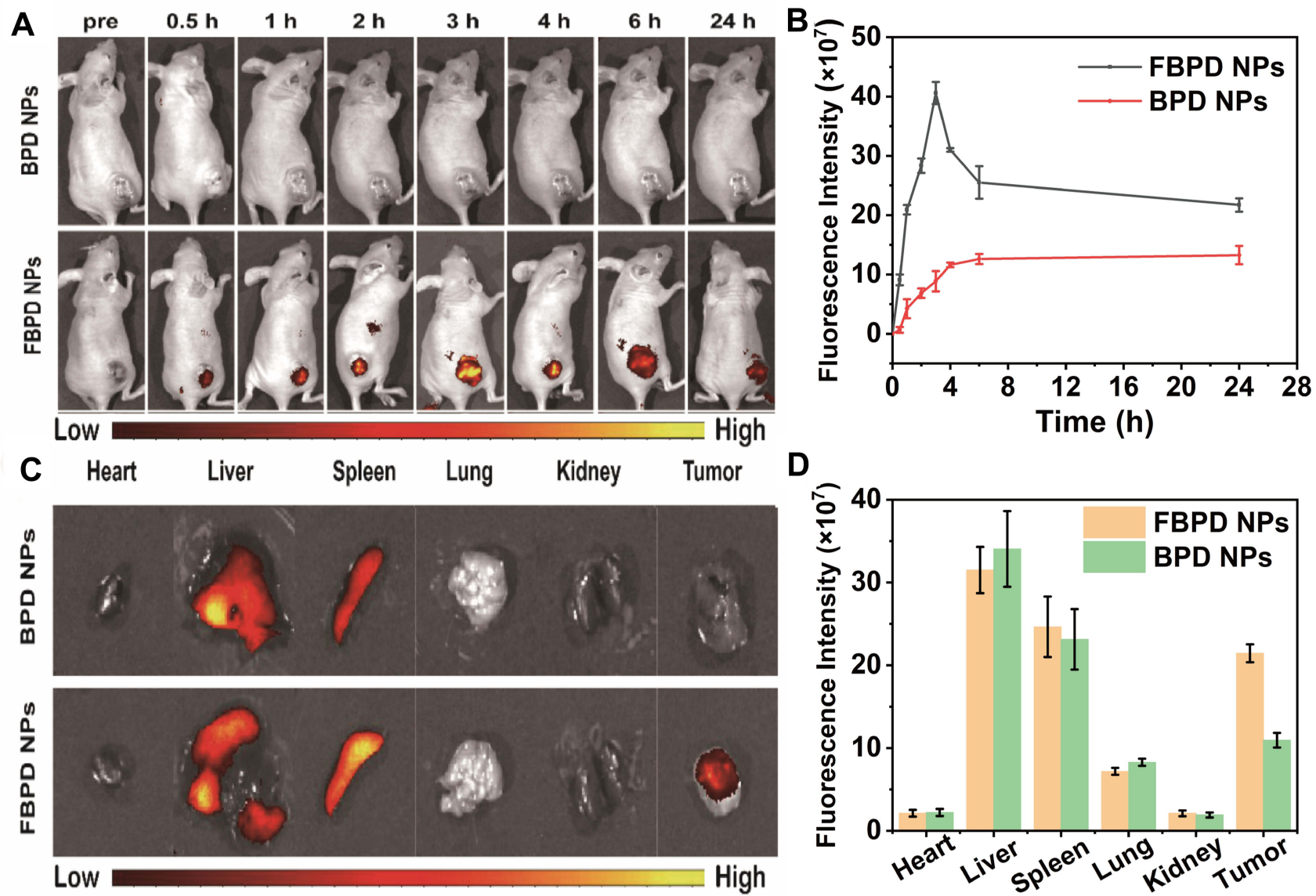

Figure 8 In vivo targeting ability. (A) In vivo fluorescence imaging and tumor targeting of a mouse at 0, 0.5, I, 2, 3, 4, 6, and 24 h post-injection of SKOV-3 tumor-bearing mice. (B) Quantitative fluorescence intensity of tumor tissue at different times $(n=3)$. (C) Bio-distribution of DiR-labeled BPD NPs or FBPD NPs in major organs excised from mice at $24 \mathrm{~h}$ post-injection. (D) Quantitative bio-distribution of BPD NPs or FBPD NPs in mice as determined by the average FL intensities of organs and tumors ( $\mathrm{n}$ $=3$ ).

treatment course for all groups, indicating no obvious histological damage in the treatment group (Figure 10B).

\section{Biosafety Assay of FBPD NPs}

In order to explored the short/long-term potential toxicity of FBPD NPs, a blood biochemical assay and H\&E staining of major organs were verified on $\mathrm{Balb} / \mathrm{c}$ mice. The result of blood biochemical revealed that negligible changes after different treatments (Figure 11A), indicating the satisfactory biocompatibility of FBPD NPs. In addition, no obvious damage to major tissues was discovered, suggesting the high therapeutic biosafety of FBPD NPs (Figure 11B).

\section{Conclusions}

In summary, we successfully constructed multifunctional theranostic FBPD NPs for highly efficient PTT/chemotherapy combination therapy with dual CT/PA imaging guidance/monitoring. The cell/tissue growth inhibition was comprehensively confirmed both in vitro and in vivo. The thermal energy generated by laser irradiation and the DOX released after the phase changed exerted a strengthened therapeutic effect on tumor cells/tissues. Dox could be rapidly released under an on-site laser irradiation, thus with reduced toxicity to non-tumor regions. In vivo experiments showed that FBPD NPs after laser irradiation could effectively inhibit the growth of tumors, 

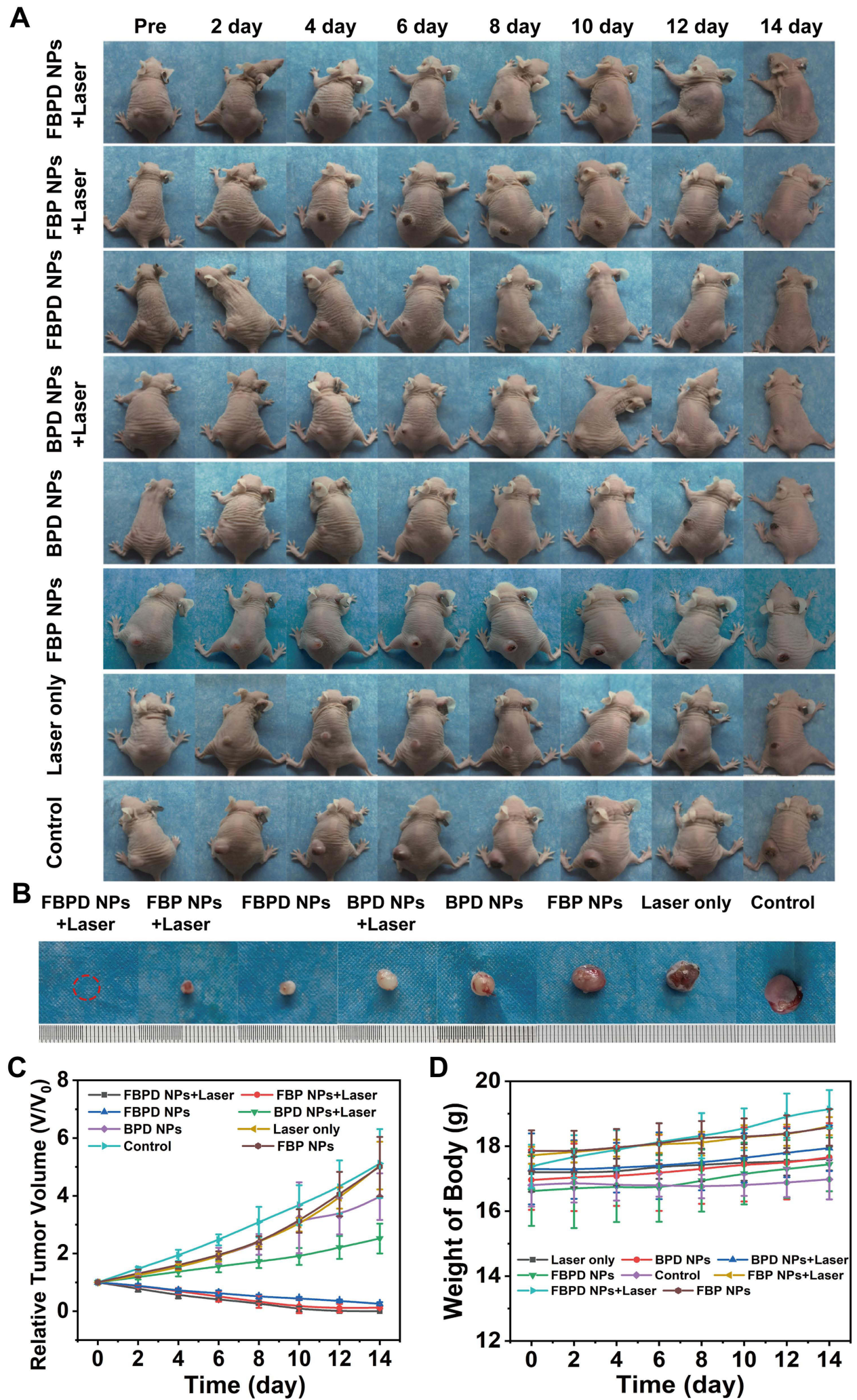

Figure 9 In vivo tumor growth inhibition. (A) Photographs of SKOV-3 tumor-bearing mice of eight groups taken during $14 \mathrm{~d}$ period after various treatments. (B) Photographs of tumors dissected from mice of eight groups after various treatments. (C) Time-dependent tumor volume curves of eight groups after various treatments ( $\mathrm{n}$ = 5). (D) Time-dependent body weights of eight groups after various treatments $(n=5)$. 


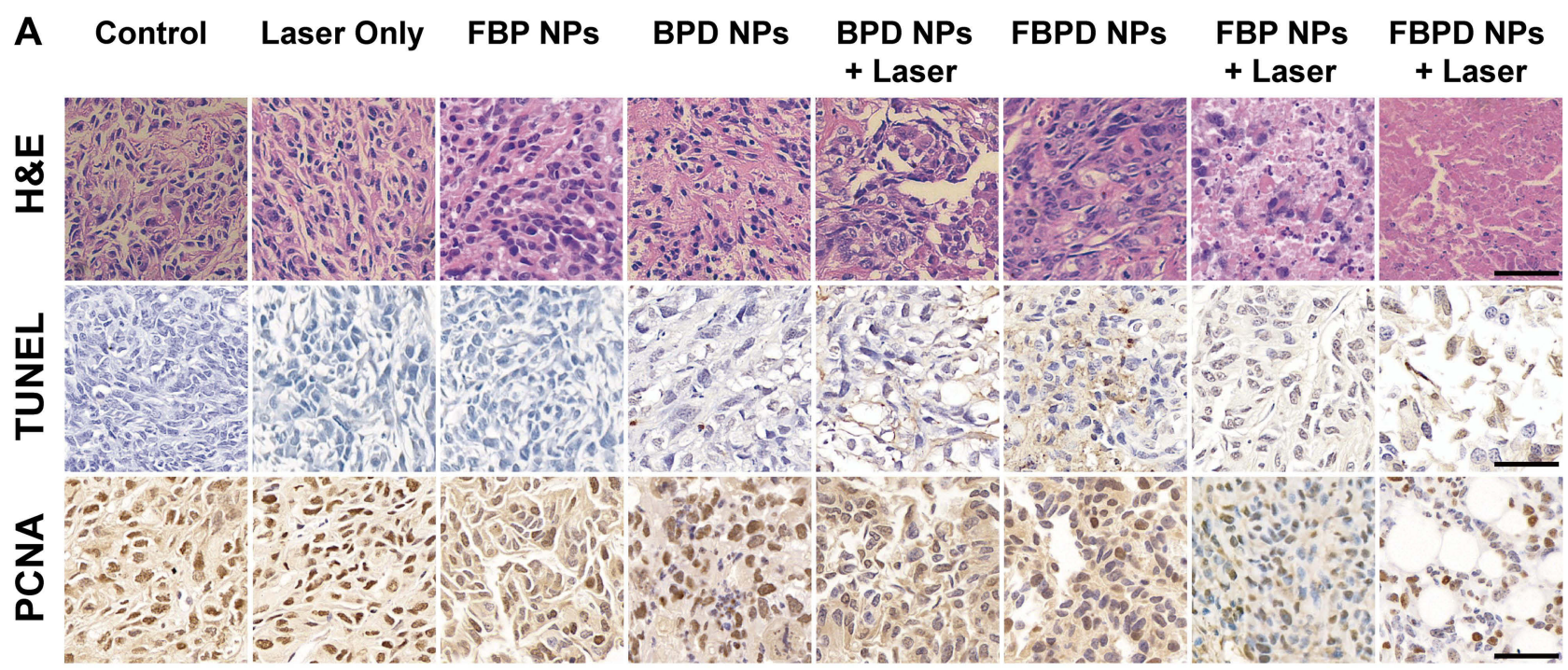

\section{B Control Laser Only FBP NPs BPD NPs BPD NPs FBPD NPs FBP NPs FBPD NPs}
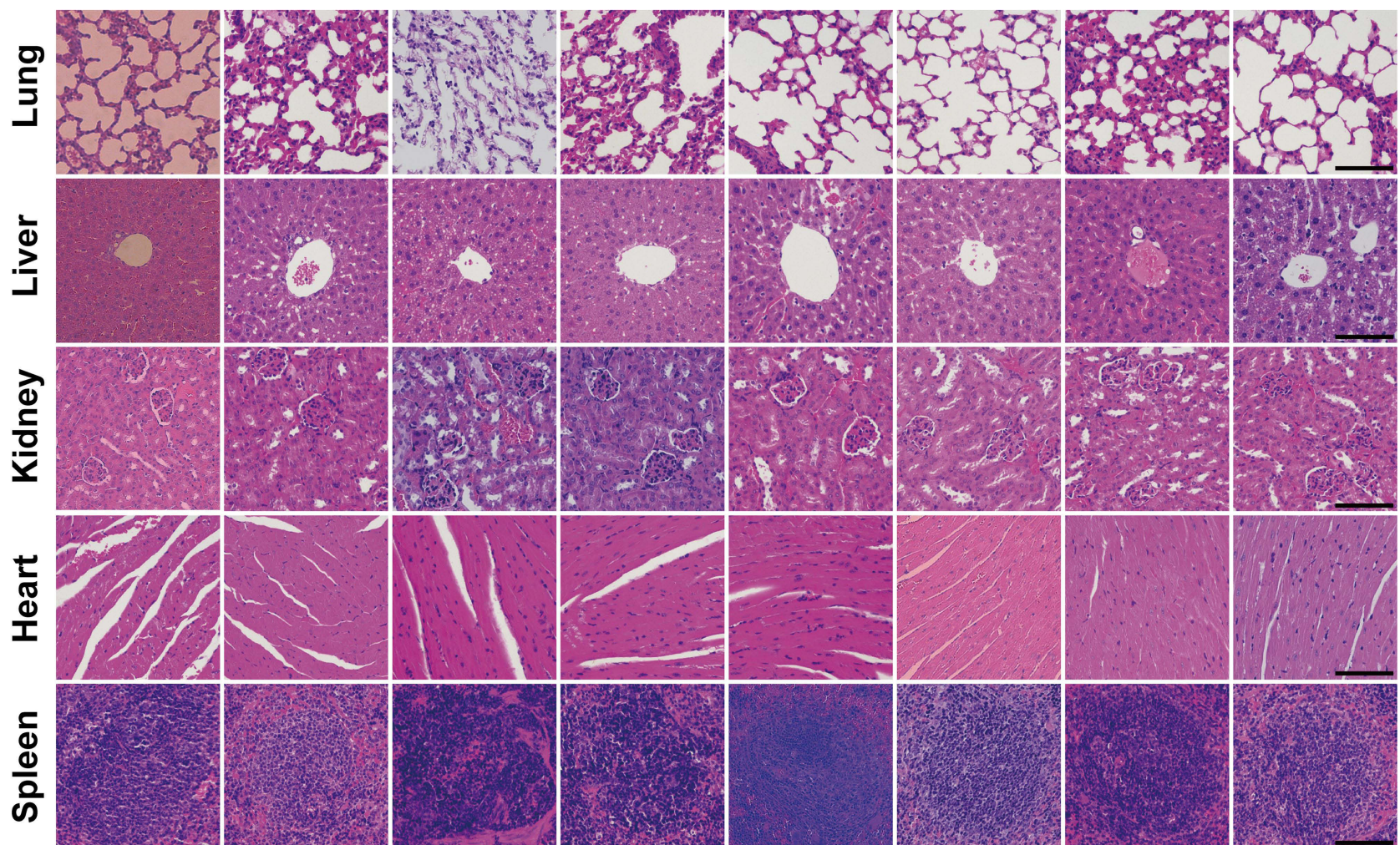

Figure 10 (A) H\&E, TUNEL and PCNA staining on tumor sections from SKOV-3 tumor-bearing mice after various treatments. (B) H\&E staining of the major organs (heart, liver, spleen, lung, and kidney) of SKOV-3 tumor-bearing mice after different treatments. The scale bars are $50 \mu \mathrm{m}$.

and the NPs effectively accumulated in tumor region assisted by FA guidance. Furthermore, these NPs enhanced $\mathrm{CT} / \mathrm{PA}$ imaging both in vitro and in vivo, offering the potential for tumor treatment guidance/monitoring. Our study provided greats insights on using FBPD NPs as a multifunctional cancer theranostics nanoplatform. 


\section{A}

$\operatorname{ALT}(10 \%)=$ AST (100 U/L) -

TBIL (10 umoL/L)-

UREA (mmoL/L) -

CREA $(10 \mu \mathrm{moL} / \mathrm{L})-$

CK (100 U/L $)-$

LDH (100 U/L) -

WBC $\left(10^{\circ} / \mathrm{L}\right)-$

Lymph $\left(10^{9} / \mathrm{L}\right)-$

Mon $\left(10^{9} / \mathrm{L}\right)$

Gran $\left(10^{9} / \mathrm{L}\right)-$

Lymph (10\%)

Mon (\%) -

Gran (10\%)

$\operatorname{RBC}\left(10^{12} / \mathrm{L}\right)-$

HGB (10 g/L) -

HCT $(10 \%)$

$\operatorname{MCV}(10 \mathrm{fL})=$ $\mathrm{MCH}(\mathrm{pg})-$

MCHC (100 g/L) RDW (\%)

PLT $\left(10^{12} / \mathrm{L}\right)-$

MPV (fL) PDW (fL)
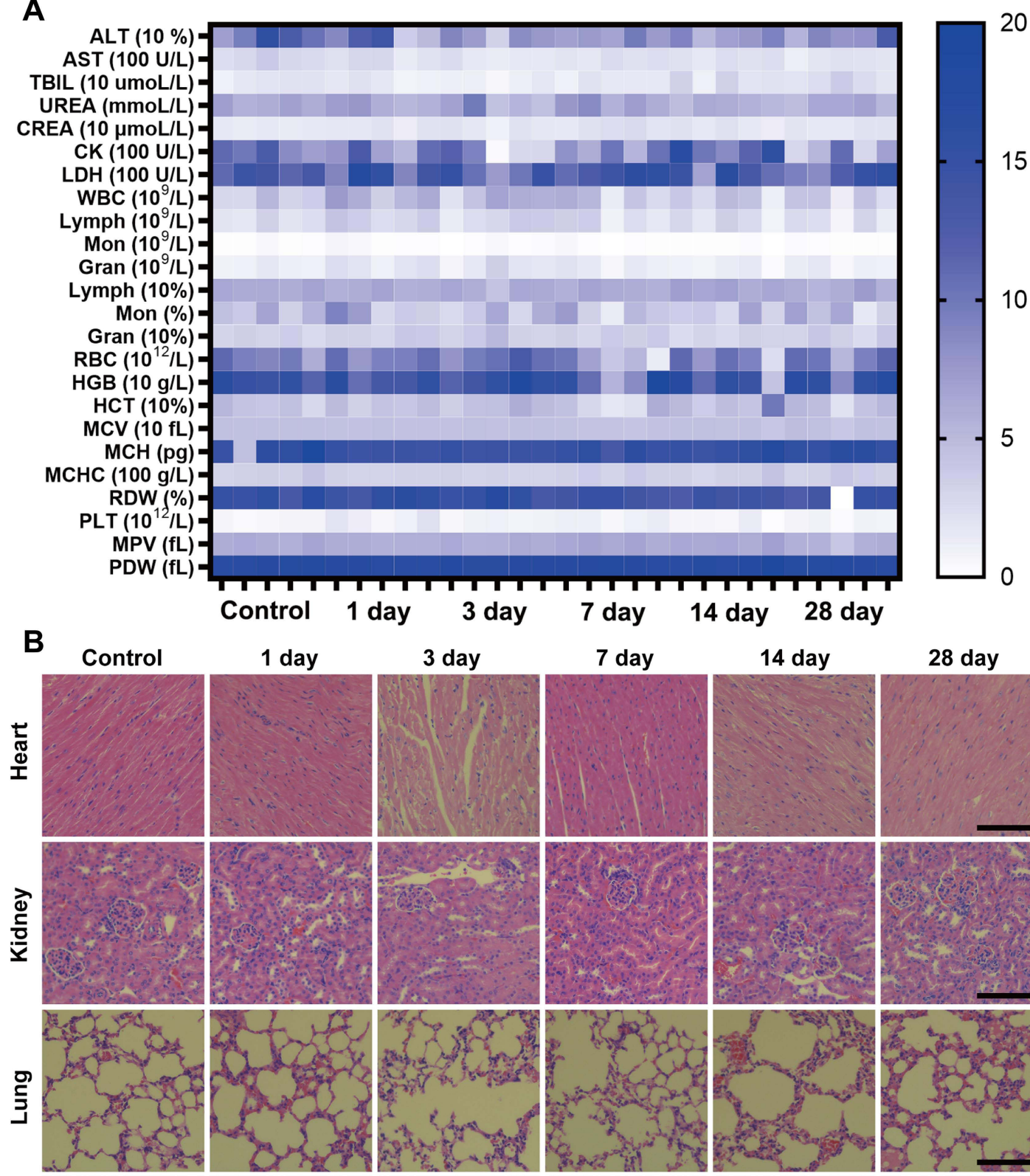

28 day
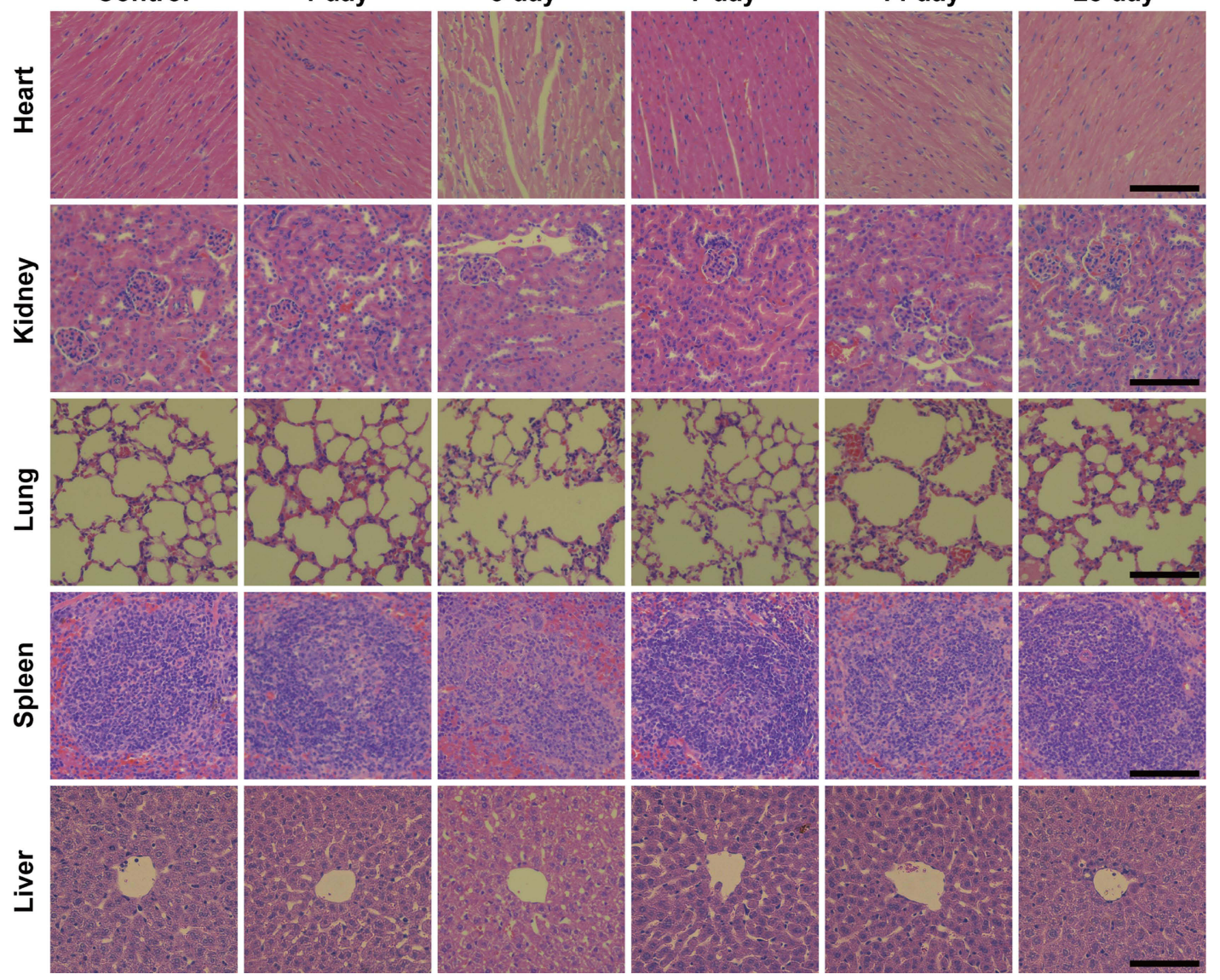

Figure II Biosafety of FBPD NPs. (A) Assay of blood index after i.v. injection of FBPD NPs $(n=5)$. (B) Images of H\&E stained slices of major organs $($ scale bar $=50 \mu m)$. 


\section{Abbreviations}

PTT, photothermal therapy; PFP, perfluoropentane; Dox, doxorubicin; FDA, Food and Drug Administration; PLGA, poly lactic-co-glycolic acid; EPR, enhanced permeation and retention; FA, folic acid; $\mathrm{Bi}_{2} \mathrm{~S}_{3}$, bismuth sulfide; CT, computed tomography; $3 \mathrm{D}$, three-dimensional; PA, photoacoustic; FBPD NPs, FA-Bi $\mathrm{S}_{3}$-PFP-Dox-PLGA nanoparticles; FBP NPs, FA-Bi ${ }_{2} \mathrm{~S}_{3}$-PFP-PLGA nanoparticles; BPD NPs, $\mathrm{Bi}_{2} \mathrm{~S}_{3}$-PFP-PLGA nanoparticles; CCK-8, Cell Counting Kit-8; DAPI, 2-(4-Amidinophenyl)-6-indolecarbamidinedihydrochloride; DiR, 1,1'-dioctadecyl-3,3,3',3'- tetramethylindotricarbocyanine iodide; $\mathrm{CHCl}_{2}$, dichloromethane; PVA, poly (vinyl alcohol); SEM, scanning electron microscopy; TEM, transmission electron microscopy; ICP-MS, inductively coupled plasma mass spectrometry; EE, encapsulation efficiency; DL, drug loading; OD, optical density; CLSM, confocal laser scanning microscopy; H\&E, Hematoxylineosin; TUNEL, TdT-mediated dUTP Nick-End Labeling; PCNA, proliferating cell nuclear antigen.

\section{Data Sharing Statement}

All data generated or analysed during this study are included in this published article.

\section{Ethics Approval and Consent to Participate}

All animal procedures were performed in accordance with the Guidelines for Care and Use of Laboratory Animals of Chongqing Medical University and approved by the Animal Ethics Committee of Chongqing Medical University.

\section{Author Contributions}

All authors made a significant contribution to the work reported, whether that is in the conception, study design, execution, acquisition of data, analysis and interpretation, or in all these areas; took part in drafting, revising or critically reviewing the article; gave final approval of the version to be published; have agreed on the journal to which the article has been submitted; and agreed to be accountable for all aspects of the work.

\section{Funding}

This work was supported by the National Natural Science Foundation of China Youth Science Foundation Project (NO.81801717), Medical Science Cultivation Fund of the First Affiliated Hospital of Chongqing Medical University
(PYJJ2018-15) and Chongqing Postgraduate Research and Innovation Project (Grant No: CYS18194).

\section{Disclosure}

The authors declare that they have no competing interests.

\section{References}

1. Wang Y, Liu X, Deng G, et al. Se@SiO2-FA-CuS nanocomposites for targeted delivery of DOX and nano selenium in synergistic combination of chemo-photothermal therapy. Nanoscale. 2018;10 (6):2866-2875. doi:10.1039/c7nr09237g

2. Bertucci A, Kim KH, Kang J, et al. Tumor-Targeting, MicroRNA-Silencing Porous Silicon Nanoparticles for Ovarian Cancer Therapy. ACS Appl Mater Interfaces. 2019;11 (27):23926-23937. doi:10.1021/acsami.9b07980

3. Luiz MT, Abriata JP, Raspantini GL, et al. In vitro evaluation of folate-modified PLGA nanoparticles containing paclitaxel for ovarian cancer therapy. Materi Sci Eng. 2019;105:110038. doi:10.1016/j. msec.2019.110038

4. Zhou Y, Hu AY, Sun AW, Zhou AB, Zhu AJ. Polyaniline-loaded $\gamma$ polyglutamic acid nanogels as a platform for photoacoustic imagingguided tumor photothermal therapy. Nanoscale. 2017;9 (34):12746-12754. doi:10.1039/c7nr04241h

5. Byrne JD, Jen YJ, Desimone JM. Use of iontophoresis for the treatment of cancer. $J$ Controlled Release. 2018;284:144-151. doi:10.1016/j.jconrel.2018.06.020

6. Grunewald T, Ledermann JA. Targeted Therapies for Ovarian Cancer. Best Pract Res Clin Obstet Gynaecol. 2017;41:139-152. doi:10.1016/ j.bpobgyn.2016.12.001

7. Harris FR, Zhang P, Yang L, et al. Targeting HER2 in patient-derived xenograft ovarian cancer models sensitizes tumors to chemotherapy. Mol Oncol. 2019;13(2):132-152. doi:10.1002/1878-0261.12414

8. Huang J, Liu F, Han X, et al. Nanosonosensitizers for Highly Efficient Sonodynamic Cancer Theranostics. Theranostics. 2018;8 (22):6178-6194. doi:10.7150/thno.29569

9. Liu P, Chen N, Yan L. Preparation, characterisation and in vitro and in vivo evaluation of CD44-targeted chondroitin sulphate-conjugated doxorubicin PLGA nanoparticles. Carbohydr Polym. 2019;213:17-26. doi:10.1016/j.carbpol.2019.02.084

10. Deshpande P, Jhaveri A, Pattni B, Biswas S, Torchilin V. Transferrin and octaarginine modified dual-functional liposomes with improved cancer cell targeting and enhanced intracellular delivery for the treatment of ovarian cancer. Drug Deliv. 2018;25(1):517-532. doi:10.1080/10717544.2018.1435747

11. Xue S, Tingting L, Zhongyuan C, et al. Luminescent/magnetic PLGA-based hybrid nanocomposites: a smart nanocarrier system for targeted codelivery and dual-modality imaging in cancer theranostics. Int J Nanomedicine. 2017;12:4299-4322. doi:10.2147/ IJN.S136766

12. Mangal S, Gao W, Tonglei LI, Zhou QT. Pulmonary delivery of nanoparticle chemotherapy for the treatment of lung cancers: challenges and opportunities. Acta Pharmacol Sin. 2017;38(6):782-797. doi:10.1038/aps.2017.34

13. Changqie $\mathrm{P}$, Yuqing L, Minyu Z, et al. Theranostic pH-sensitive nanoparticles for highly efficient targeted delivery of doxorubicin for breast tumor treatment. Int $J$ Nanomedicine. 2018;13:1119-1137. doi:10.2147/IJN.S147464

14. Gao Z, Liu X, Deng G, Zhou F, Lu PJ. Fe3O4@mSiO2-FA-CuS-PEG nanocomposites for magnetic resonance imaging and targeted chemo-photothermal synergistic therapy of cancer cells. Dalton Transactions. 2016;45(34):13456-13465. doi:10.1039/c6dt01714b 
15. Shuxin S, Ying L, Yunbin X, et al. Folate-conjugated nanobubbles selectively target and kill cancer cells via ultrasound-triggered intracellular explosion. Biomaterials. 2018;181:293-306. doi:10.1016/j. biomaterials.2018.07.030

16. Zhong Y, Su T, Shi Q, Feng Y, Yang H. Co-Administration Of iRGD Enhances Tumor-Targeted Delivery And Anti-Tumor Effects Of Paclitaxel-Loaded PLGA Nanoparticles For Colorectal Cancer Treatment. Int J Nanomedicine. 2019;14:8543-8560. doi:10.2147/ IJN.S219820

17. Li X, Jiang X. Microfluidics for producing poly (lactic- co -glycolic acid)-based pharmaceutical nanoparticles. Adv Drug Deliv Rev. 2018;128:101-114. doi:10.1016/j.addr.2017.12.015

18. Cao Y, He J, Liu J, Zhang M, Ni P. Folate-Conjugated Polyphosphoester with Reversible Cross-Linkage and Reduction-Sensitivity for Drug Delivery. ACS Appl Mater Interfaces. 2018;10(9):7811-7820. doi:10.1021/acsami.7b18887

19. Tang Y, Li Y, Xu R, Li S, Li Z. Self-assembly of folic acid dextran conjugates for cancer chemotherapy. Nanoscale. 2018;10 (36):17265-17274. doi:10.1039/c8nr04657c

20. Zang Y, Wei Y, Shi Y, Chen Q, Xing D. Chemo/Photoacoustic Dual Therapy with mRNA-Triggered DOX Release and Photoinduced Shockwave Based on a DNA-Gold Nanoplatform. Small. 2016;12 (6):756-769. doi:10.1002/smll.201502857

21. Niu C, Xu Y, An S, et al. loaded PLGA nanoparticles for photothermal tumor ablation. Sci Rep. 2017;7(1):5490. doi:10.1038/s41598017-06122-1

22. Zhang N, Song J, Liu Y, Liu M, Yang Z. Photothermal therapy mediated by phase-transformation nanoparticles facilitates delivery of anti-PD1 antibody and synergizes with antitumor immunotherapy for melanoma. J Control Release. 2019;306:15-28. doi:10.1016/j. jconrel.2019.05.036

23. Lei P, An R, Zheng X, et al. Ultrafast synthesis of ultrasmall polyethylenimine-protected $\mathrm{AgBiS}_{2}$ nanodots by "rookie method" for in vivo dual-modal CT/PA imaging and simultaneous photothermal therapy. Nanoscale. 2018;10(35):16765-16774. doi:10.1039/ c8nr04870c

24. Zhang L, Wang D, Yang K, et al. Mitochondria-Targeted Artificial "Nano-RBCs" for Amplified Synergistic Cancer Phototherapy by a Single NIR Irradiation. Adv Sci. 2018;5(8):1800049. doi:10.1002/ advs.201800049

25. Li WT, Peng JR, Tan LW, Wu J, Qian ZY. Mild Photothermal Therapy/Photodynamic Therapy/Chemotherapy of Breast Cancer by Lyp-1 Modified Docetaxel/IR820 Co-loaded Micelles. Biomaterials. 2016;106:119-133. doi:10.1016/j.biomaterials.2016.08.016

26. Chen L, Zhou L, Wang C, Han Y, Dong C. Tumor-targeted Drug and CpG Delivery System for Phototherapy and Docetaxelenhanced Immunotherapy with Polarization toward M1-type Macrophages on Triple Negative Breast Cancers. Adv Mater. 2019;31(52):e1904997. doi:10.1002/adma.201904997

27. Cheng L, He W, Gong H, et al. PEGylated Micelle Nanoparticles Encapsulating a Non-Fluorescent Near-Infrared Organic Dye as a Safe and Highly-Effective Photothermal Agent for In Vivo Cancer Therapy. Adv Funct Mater. 2013;23(47):5893-5902. doi:10.1002/adfm.201301045

28. Meng Z, Wei F, Wang R, et al. NIR-Laser-Switched In Vivo Smart Nanocapsules for Synergic Photothermal and Chemotherapy of Tumors. Adv Mater. 2016;28(2):245-253. doi:10.1002/ adma.201502669

29. Zhang D, Cui P, Dai Z, et al. Tumor microenvironment responsive $\mathrm{FePt} / \mathrm{MoS} 2$ nanocomposites with chemotherapy and photothermal therapy for enhancing cancer immunotherapy. Nanoscale. 2019;11 (42):19912-19922. doi:10.1039/c9nr05684j

30. Chen Y, Zhao G, Wang S, He YW, Wang J. Platelet-membranecamouflaged bismuth sulfide nanorods for synergistic radio-photothermal therapy against cancer. Biomater Sci. 2019;7 (8):3450-3459. doi:10.1039/c9bm00599d
31. Yang $M$, Zhang N, Zhang $T$, Yin $X$, Shen J. Fabrication of doxorubicin-gated mesoporous polydopamine nanoplatforms for multimode imaging-guided synergistic chemophotothermal therapy of tumors. Drug Deliv. 2020;27(1):367-377. doi:10.1080/ 10717544.2020.1730523

32. Zhang HK, Chen Y, Kang J, Lisok A, Boctor EM. Prostate-specific membrane antigen-targeted photoacoustic imaging of prostate cancer in vivo. $J$ Biophotonics. 2018;11(9):e201800021. doi:10.1002/ jbio.201800021

33. Liu J, Zheng X, Yan L, et al. Bismuth sulfide nanorods as a precision nanomedicine for in vivo multimodal imaging-guided photothermal therapy of tumor. ACS Nano. 2015;9(1):696-707. doi:10.1021/ nn506137n

34. Wang X, Ai A, Yu Z, Deng M, Wang X. Dual-modal non-invasive imaging in vitro and in vivo monitoring degradation of PLGA scaffold based gold nanoclusters. Mater Sci Eng C Mater Biol Appl. 2020;107:110307. doi:10.1016/j.msec.2019.110307

35. Zhou D, Li C, He M, et al. Folate-targeted perfluorohexane nanoparticles carrying bismuth sulfide for use in US/CT dual-mode imaging and synergistic high-intensity focused ultrasound ablation of cervical cancer. J Mater Chem B. 2016;4(23):4164-4181. doi:10.1039/ c6tb00261g

36. Chen S, Liu Y, Zhu S. Dual-mode imaging and therapeutic effects of drug-loaded phase-transition nanoparticles combined with near-infrared laser and low-intensity ultrasound on ovarian cancer. Drug Deliv. 2018;25(1):1683-1693. doi:10.1080/ 10717544.2018.1507062

37. Xiong J, Feng J, Qiu L, et al. SDF-1-loaded PLGA nanoparticles for the targeted photoacoustic imaging and photothermal therapy of metastatic lymph nodes in tongue squamous cell carcinoma. Int J Pharm. 2019;554:93-104. doi:10.1016/j.ijpharm.2018.10.064

38. Liming D, Xiaojun C, Danli S, et al. A Laser-Activated Biocompatible Theranostic Nanoagent for Targeted Multimodal Imaging and Photothermal Therapy. Theranostics. 2017;7 (18):4410-4423. doi:10.7150/thno.21283

39. Bazylińska U, Kulbacka J, Chodaczek G. Nanoemulsion Structural Design in Co-Encapsulation of Hybrid Multifunctional Agents: influence of the Smart PLGA Polymers on the Nanosystem-Enhanced Delivery and Electro-Photodynamic Treatment. Pharmaceutics. 2019;11(8):405. doi:10.3390/pharmaceutics 11080405

40. Zhang M, Xu C, Liu D, Han MK, Wang L, Merlin D. Oral delivery of nanoparticles loaded with ginger active compound, 6-shogaol, attenuates ulcerative colitis and promotes wound healing in a murine model of ulcerative colitis. J Crohns Colitis. 2018;12(2):217-229. doi:10.1093/ecco-jcc/jjx115

41. Yuxin L, Luoyuan L, Quanwei G, et al. Novel Cs-Based Upconversion Nanoparticles as Dual-Modal CT and UCL Imaging Agents for Chemo-Photothermal Synergistic Therapy. Theranostics. 2016;6(10):1491-1505. doi:10.7150/thno.15111

42. Qiu J, Xiao Q, Zheng X. Single W18O49 nanowires: a multifunctional nanoplatform for computed tomography imaging and photothermal/photodynamic/radiation synergistic cancer therapy. Nano Res. 2015;11:3580-3590. doi:10.1007/s12274-015-0858-Z

43. Li Z, Hu Y, Chang M, et al. Highly porous PEGylated Bi2S3 nano-urchins as a versatile platform for in vivo triple-modal imaging, photothermal therapy and drug delivery. Nanoscale. 2016;8 (35):16005-16016. doi:10.1039/c6nr03398a

44. Mohammadi Gazestani A, Khoei S, Khoee S, Emamgholizadeh Minaei S, Motevalian M. In vivo evaluation of the combination effect of near-infrared laser and 5-fluorouracil-loaded PLGA-coated magnetite nanographene oxide. Artif Cells Nanomed Biotechnol. 2018;46 (sup2):25-33. doi:10.1080/21691401.2018.1450265

45. Yang P, Bi H, Dai Y, et al. CuS-Pt (IV)-PEG-FA nanoparticles for targeted photothermal and chemo-therapy. J Mater Chem B. 2016;4 (35):5938-5946. doi:10.1039/c6tb01540a 


\section{Publish your work in this journal}

The International Journal of Nanomedicine is an international, peerreviewed journal focusing on the application of nanotechnology in diagnostics, therapeutics, and drug delivery systems throughout the biomedical field. This journal is indexed on PubMed Central, MedLine, CAS, SciSearch ${ }^{\mathbb{R}}$, Current Contents ${ }^{\mathbb{B}} /$ Clinical Medicine,
Journal Citation Reports/Science Edition, EMBase, Scopus and the Elsevier Bibliographic databases. The manuscript management system is completely online and includes a very quick and fair peer-review system, which is all easy to use. Visit http://www.dovepress.com/ testimonials.php to read real quotes from published authors.

Submit your manuscript here: https://www.dovepress.com/international-journal-of-nanomedicine-journal 